\title{
Analytical State-Average Complete-Active-Space Self-Consistent Field Nonadiabatic Coupling Vectors: Implementation with Density-Fitted Two-Electron Integrals and Application to Conical Intersections
}

\author{
Ignacio Fdez. Galván Mickaël G. Delcey Thomas Bondo Pedersen \\ Francesco Aquilante Roland Lindh
}

Initial geometries, final optimized geometries and branching vectors $(\hat{x}$ and $\hat{y})$. All coordinates in $\AA$.

\section{S1 Ethylene (a)}
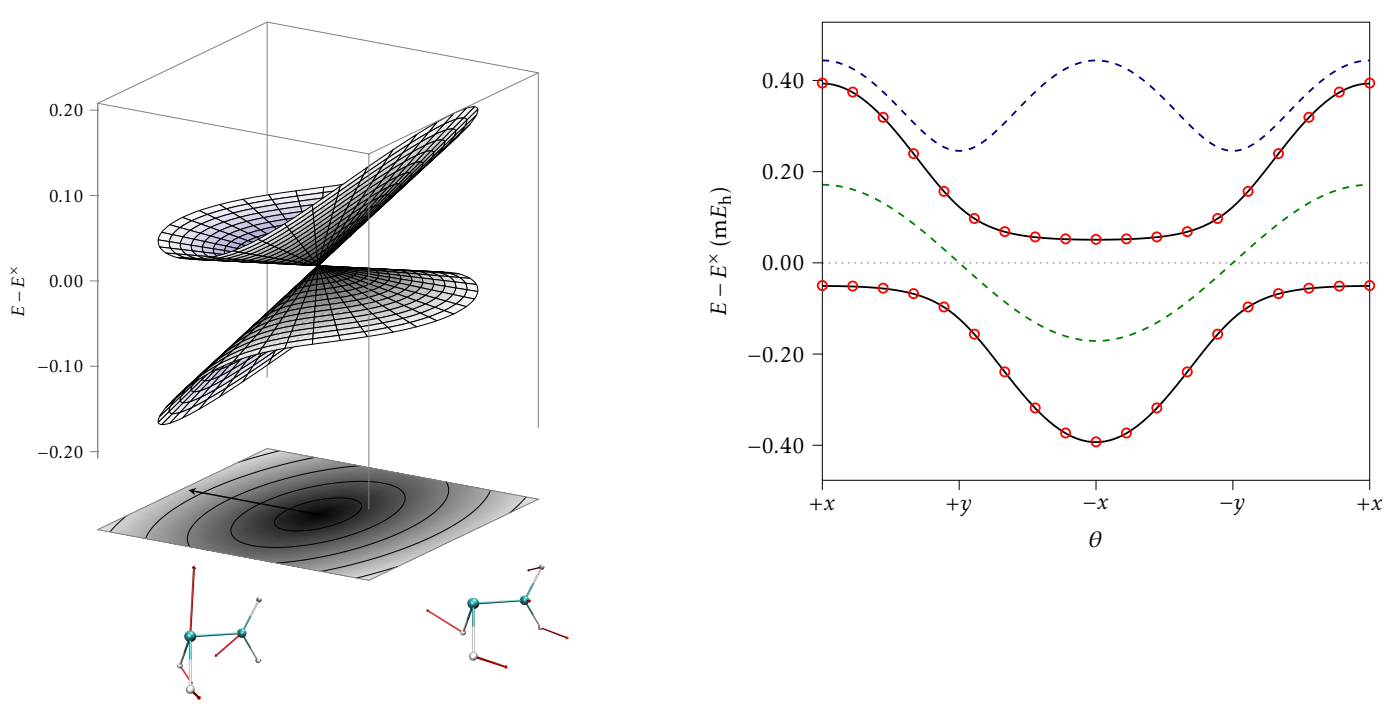

$\begin{array}{rrrr}\text { C } & 0.00000 & 0.00000 & 0.00000 \\ \text { C } & 1.41648 & 0.00000 & 0.00000 \\ \text { Initial geometry: H } & -0.54199 & 0.00000 & -0.93255 \\ \text { H } & 1.95847 & 0.59943 & 0.71438 \\ \text { H } & -0.54199 & 0.00000 & 0.93255 \\ \text { H } & 1.95847 & -0.59943 & 0.71438\end{array}$




\section{S1.1 ANO-RCC-VDZP}

$\begin{array}{rrrrr} & \text { C } & 0.12224852 & 0.00001916 & 0.00222387 \\ & \text { C } & 1.50997482 & 0.00018222 & -0.12190895 \\ \text { Optimized geometry: } & \text { H } & -0.53465672 & -0.00016927 & -0.85910430 \\ & \text { H } & 1.78615927 & 0.69322745 & 0.73193307 \\ \text { H } & -0.42007957 & 0.00002555 & 0.94383446 \\ & \text { H } & 1.78579368 & -0.69328510 & 0.73178183\end{array}$

\begin{tabular}{|c|c|c|c|c|}
\hline \multirow{6}{*}{$\hat{x}$ vector: } & C & 0.435700015 & -0.000052319 & 0.240161591 \\
\hline & C & -0.152729031 & 0.000248070 & -0.725573747 \\
\hline & $\mathrm{H}$ & -0.008184547 & 0.000013092 & -0.011920075 \\
\hline & $\mathrm{H}$ & -0.100877895 & -0.169134545 & 0.24442845 \\
\hline & $\mathrm{H}$ & -0.072809780 & -0.000093998 & 0.008347732 \\
\hline & $\mathrm{H}$ & -0.101098762 & 0.169019699 & 0.24455604 \\
\hline \multirow{6}{*}{$\hat{y}$ vector: } & C & -0.000249995 & -0.142432530 & $-0.0003625 \mathrm{~g}$ \\
\hline & C & -0.000005347 & -0.007272702 & 0.00010466 \\
\hline & $\mathrm{H}$ & -0.000071394 & 0.336976396 & -0.000019385 \\
\hline & $\mathrm{H}$ & 0.382904134 & 0.149488216 & -0.381568106 \\
\hline & $\mathrm{H}$ & 0.000133263 & -0.486660722 & 0.00004217 \\
\hline & & -0.382710661 & 0.149901341 & 0.381803246 \\
\hline
\end{tabular}

\section{S1.2 ANO-RCC-VTZP}

$\begin{array}{rrrrr} & \text { C } & 0.11785473 & 0.00002372 & 0.00242448 \\ \text { Optimized geometry: } & \text { H } & 1.50343345 & 0.00004222 & -0.11686378 \\ & \text { H } & 1.73199918 & -0.00004773 & -0.86054458 \\ & \text { H } & -0.42334133 & 0.69292121 & 0.73120866 \\ & \text { H } & 1.79170886 & -0.00000212 & 0.94134666 \\ & & & \end{array}$

\begin{tabular}{|c|c|c|c|c|}
\hline \multirow{6}{*}{$\hat{x}$ vector: } & C & 0.431985914 & -0.000162484 & 0.238841626 \\
\hline & C & -0.166934546 & 0.000100762 & -0.727783721 \\
\hline & $\mathrm{H}$ & -0.007067399 & 0.000079950 & -0.015100690 \\
\hline & $\mathrm{H}$ & -0.093422855 & -0.164116857 & 0.246961732 \\
\hline & $\mathrm{H}$ & -0.071032426 & -0.000018973 & 0.010001237 \\
\hline & $\mathrm{H}$ & -0.093528687 & 0.164117602 & 0.247079816 \\
\hline \multirow{6}{*}{$\hat{y}$ vector: } & C & -0.000107423 & -0.1515205 & -0.00 \\
\hline & C & 0.000037960 & 0.006757974 & 0.00009948 \\
\hline & $\mathrm{H}$ & -0.000014896 & 0.339334474 & -0.000006706 \\
\hline & $\mathrm{H}$ & 0.380687748 & 0.143055711 & -0.38733316 \\
\hline & $\mathrm{H}$ & 0.000029484 & -0.480697537 & -0.00001955 \\
\hline & & -0.380632873 & 0.143069880 & 0.387314321 \\
\hline
\end{tabular}




\section{S1.3 ANO-RCC-VQZP}

$\begin{array}{rrrrr} & \text { C } & 0.11815911 & -0.00000110 & 0.00285155 \\ & \text { C } & 1.50289536 & -0.00001562 & -0.11690234 \\ \text { Optimized geometry: } & \text { H } & -0.53224553 & 0.00001412 & -0.85944705 \\ & \text { H } & 1.79184006 & 0.69243679 & 0.73046042 \\ \text { H } & -0.42307984 & -0.00000237 & 0.94132412 \\ & \text { H } & 1.79187084 & -0.69243182 & 0.73047330\end{array}$

\begin{tabular}{|c|c|c|c|c|}
\hline & C & 0.433257879 & 0.000018162 & 0.238895169 \\
\hline & C & -0.167546470 & -0.000030813 & -0.726977128 \\
\hline & $\mathrm{H}$ & -0.007803185 & -0.000033703 & -0.015717985 \\
\hline & $\mathrm{H}$ & -0.093171345 & -0.164365429 & 0.246736029 \\
\hline & $\mathrm{H}$ & -0.071672006 & 0.000045326 & 0.010399775 \\
\hline & $\mathrm{H}$ & -0.093064872 & 0.164366457 & 0.246664141 \\
\hline & C & 0.000057215 & -0.153290061 & -0.000002725 \\
\hline & C & -0.000026604 & 0.007669817 & -0.000059978 \\
\hline & $\mathrm{H}$ & 0.000042885 & 0.340021076 & 0.000008944 \\
\hline & $\mathrm{H}$ & 0.380139415 & 0.142660410 & -0.387799825 \\
\hline & $\mathrm{H}$ & 0.000001433 & -0.479810701 & 0.000008701 \\
\hline & H & -0.380214343 & 0.142749460 & 0.38784488 \\
\hline
\end{tabular}


S2 Ethylene (b)

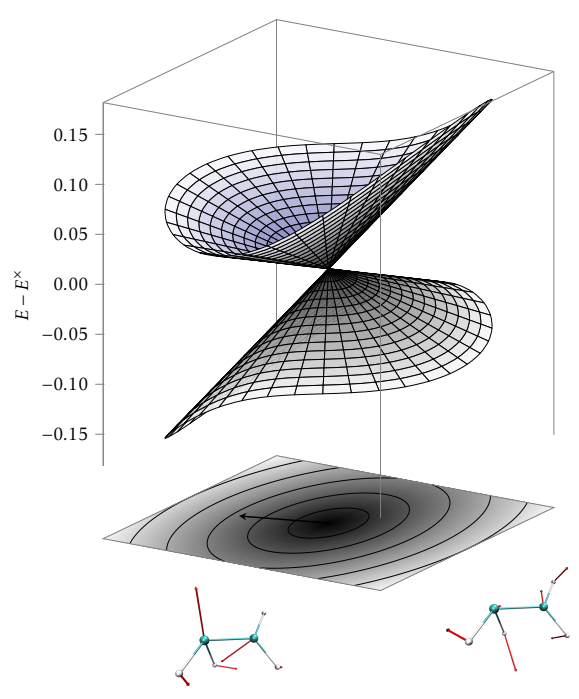

C $\quad 0.000000$

C $\quad 0.000000$

C $\quad 0.943102$

Initial geometry:

$$
\mathrm{H} \quad-0.939150
$$

H $\quad 0.262249$

H $\quad 0.565287$

0.000000
0.000000

0.100000

$-0.099581$

$-0.911409$

0.946139

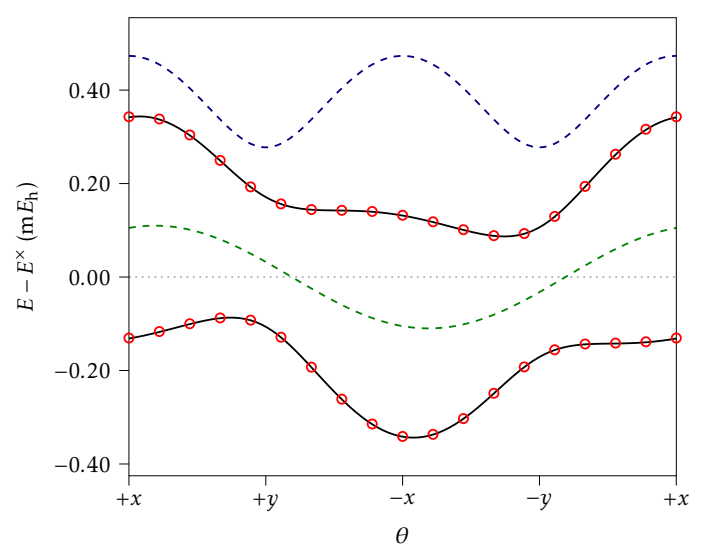

0.725000

$-0.725000$

1. 269500

1. 276363

$-1.269500$

$-0.569500$

$\begin{array}{rrrrr} & \text { C } & -0.03169250 & -0.07561723 & 0.69767616 \\ & \text { C } & -0.19603066 & -0.15607371 & -0.69862041 \\ \text { Optimized geometry: } & \text { H } & 0.92540693 & -0.03041899 & 1.21195234 \\ & \text { H } & -0.86237535 & 0.17603623 & 1.34412652 \\ \text { H } & 0.54463858 & -0.68432192 & -1.29254799 \\ \text { H } & 0.45154100 & 0.80554462 & -0.55572362\end{array}$

\begin{tabular}{|c|c|c|c|c|}
\hline \multirow{6}{*}{$\hat{x}$ vector: } & $\mathrm{C}$ & 0.225048939 & 0.254011725 & -0.522658802 \\
\hline & C & -0.531379744 & -0.357812912 & 0.026010291 \\
\hline & $\mathrm{H}$ & 0.015592821 & -0.021998361 & 0.070832688 \\
\hline & $\mathrm{H}$ & -0.003164225 & 0.024248805 & 0.004363938 \\
\hline & $\mathrm{H}$ & 0.150502433 & 0.115184524 & 0.054604714 \\
\hline & $\mathrm{H}$ & 0.143399776 & -0.013633780 & 0.36 \\
\hline \multirow{6}{*}{$\hat{y}$ vector: } & C & -0.307385799 & 0.131645 & 0.0 \\
\hline & C & -0.029778295 & -0.005679907 & 0.099605477 \\
\hline & $\mathrm{H}$ & 0.019888829 & 0.438901597 & -0.08550281 \\
\hline & $\mathrm{H}$ & -0.045578296 & -0.335614923 & 0.09528247 \\
\hline & $\mathrm{H}$ & -0.193871367 & 0.118793490 & -0.24675047 \\
\hline & & 0.556724928 & -0.348046212 & 0.061218953 \\
\hline
\end{tabular}



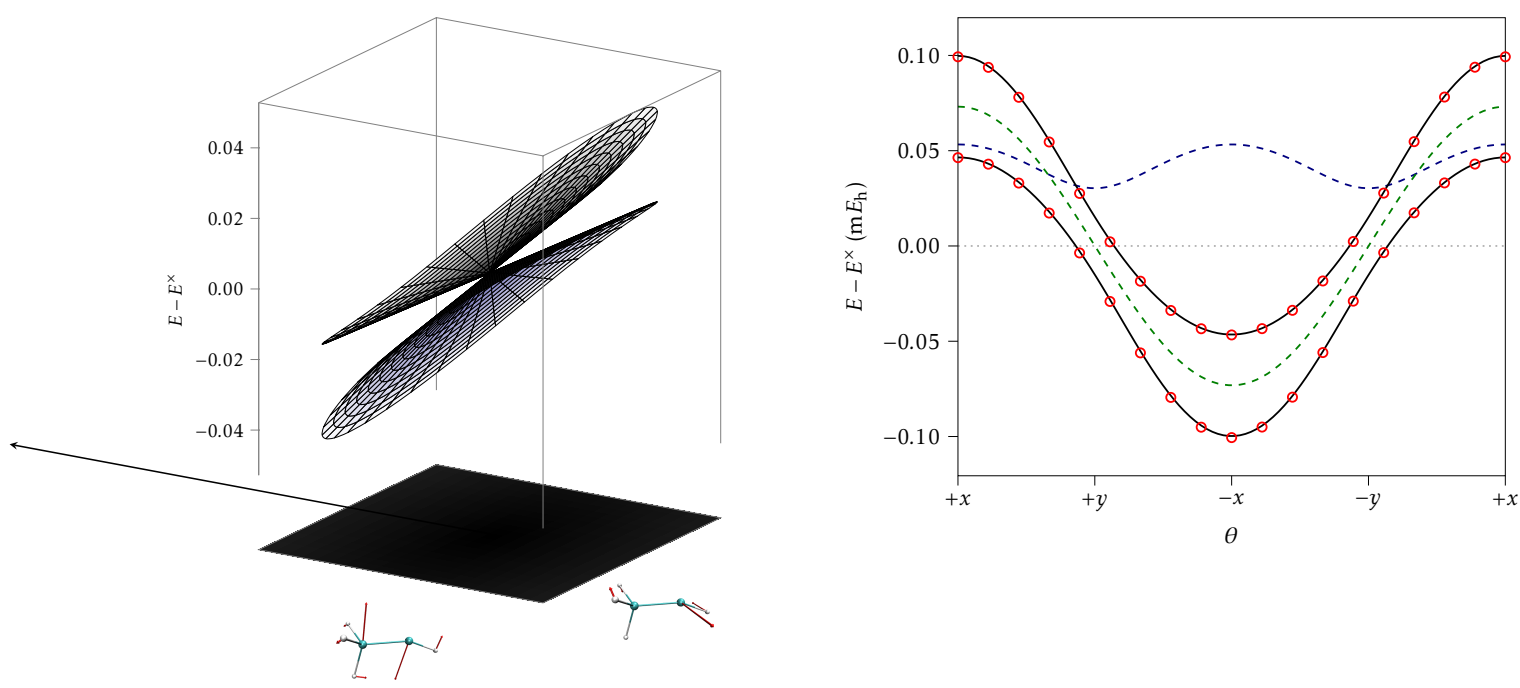

$\begin{array}{rrrr}\text { Initial geometry: } & 0.771060 & -0.000000 & 0.000001 \\ \text { C } & -0.771060 & -0.000000 & -0.000001 \\ \text { H } & -1.156219 & 0.877740 & -0.506761 \\ \text { H } & -1.156221 & -0.000000 & 1.013529 \\ \text { H } & -1.156219 & -0.877740 & -0.506761 \\ & 1.156221 & 0.716673 & 0.716673\end{array}$

$\begin{array}{rrrrr} & \text { C } & 0.60889574 & 0.30038555 & 0.15017132 \\ & \text { C } & -0.81645795 & 0.03725047 & -0.01358461 \\ \text { Optimized geometry: } & \text { H } & -1.33635759 & 0.82163281 & -0.56096310 \\ & \text { H } & -1.26402930 & -0.01262876 & 0.98352333 \\ \text { H } & -1.02276322 & -0.91095560 & -0.50712669 \\ \text { H } & 1.51827433 & 0.48098853 & 0.66465975\end{array}$

$\hat{x}$ vector: $\begin{array}{rrrr}\mathrm{C} & -0.284729133 & -0.030131991 & 0.658320697 \\ \mathrm{H} & -0.082056333 & 0.009272210 & -0.571782963 \\ \mathrm{H} & 0.189860237 & 0.004817481 & 0.037675834 \\ \mathrm{H} & -0.081543876 & -0.037289892 & 0.079995270 \\ \mathrm{H} & 0.113038560 & 0.012322060 & -0.0343157603\end{array}$

$\hat{y}$ vector: $\begin{array}{rrrr}\mathrm{C} & -0.142276026 & 0.791116733 & -0.025605111 \\ \mathrm{H} & -0.030390654 & -0.171054616 & 0.005343769 \\ \mathrm{H} & 0.008566645 & -0.036698282 & -0.137746451 \\ \mathrm{H} & 0.031918667 & -0.045470011 & 0.001886254 \\ \mathrm{H} & 0.093883744 & -0.519836979 & 0.139122138 \\ & & \end{array}$ 
S4 Methaniminium (d)
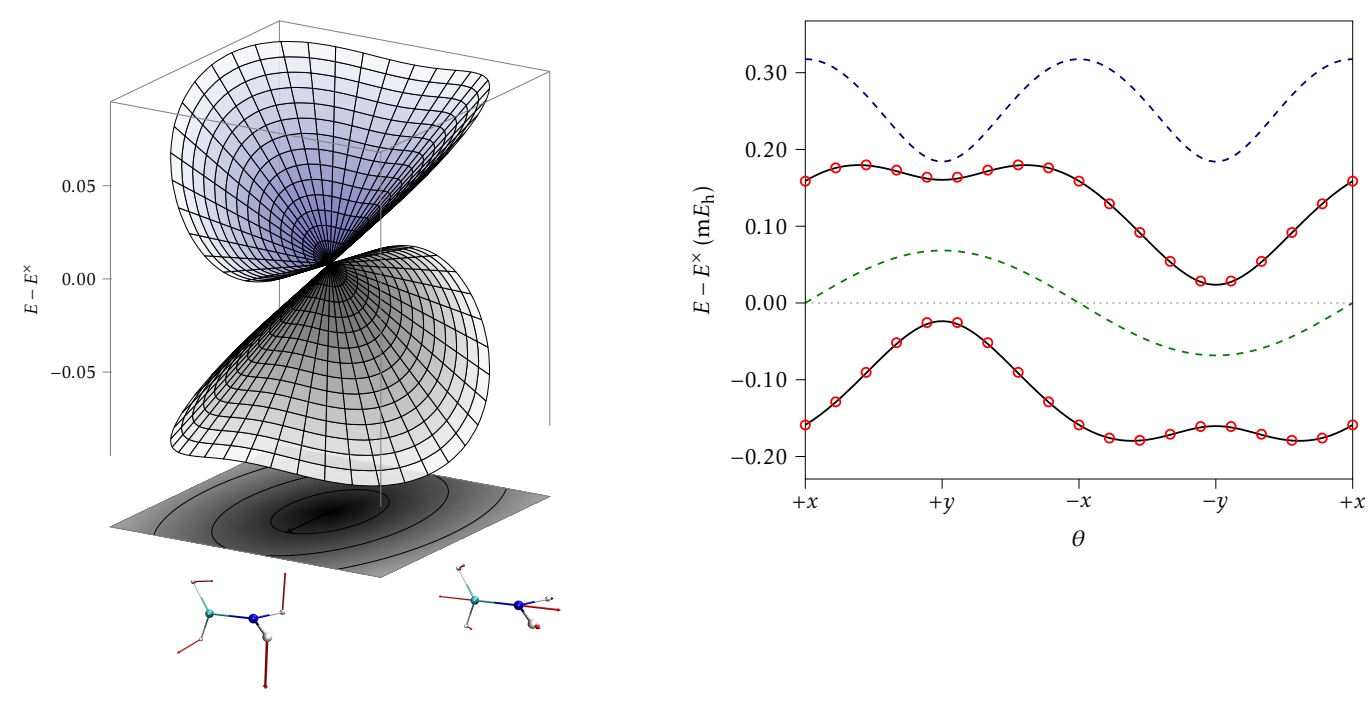

$\begin{array}{rrrr}\mathrm{C} & 0.00000 & 0.00000 & 0.00000 \\ \mathrm{~N} & 1.29450 & 0.00000 & 0.00000 \\ \text { Initial geometry: } & -0.57034 & 0.00000 & -0.93388 \\ \mathrm{H} & 1.84217 & -0.86059 & -0.01502 \\ \mathrm{H} & -0.57032 & -0.00000 & 0.93390 \\ \mathrm{H} & 1.84217 & 0.86059 & 0.01502\end{array}$

$\begin{array}{rrrrr} & \text { C } & -0.05833241 & -0.00000005 & 0.00000641 \\ \text { ON } & 1.31445906 & 0.00000006 & -0.00000028 \\ \text { Optimized geometry: } & \text { H } & -0.56379787 & 0.00745483 & -0.95365092 \\ & \text { H } & 1.85481909 & -0.85720608 & -0.00670335 \\ \text { H } & -0.56378702 & -0.00745494 & 0.95366971 \\ \text { H } & 1.85481916 & 0.85720618 & 0.00669843\end{array}$

\begin{tabular}{|c|c|c|c|c|}
\hline \multirow{6}{*}{$\hat{x}$ vector: } & C & 0.000010386 & 0.000000529 & 0.000000132 \\
\hline & $\mathrm{N}$ & 0.000025318 & -0.000001100 & -0.000000052 \\
\hline & $\mathrm{H}$ & 0.000007463 & -0.472627807 & -0.003689252 \\
\hline & $\mathrm{H}$ & -0.000027383 & 0.004118222 & -0.52592024 \\
\hline & $\mathrm{H}$ & 0.000007414 & 0.472628449 & 0.003689206 \\
\hline & $\mathrm{r}$ & -0.000023198 & -0.004118292 & 0.52592020 \\
\hline \multirow{6}{*}{$\hat{y}$ vector: } & C & -0.861262270 & -0.000000230 & -0.0 \\
\hline & $\mathrm{N}$ & 0.451880545 & 0.000000011 & 0.00000005 \\
\hline & $\mathrm{H}$ & 0.133751817 & 0.000328374 & -0.042080118 \\
\hline & $\mathrm{H}$ & 0.070938088 & -0.048193011 & -0.00037670 \\
\hline & $\mathrm{H}$ & 0.133753743 & -0.000328218 & 0.04208165 \\
\hline & & 0.070938078 & 0.048193074 & 0.00037603 \\
\hline
\end{tabular}


S5 Methaniminium (e)
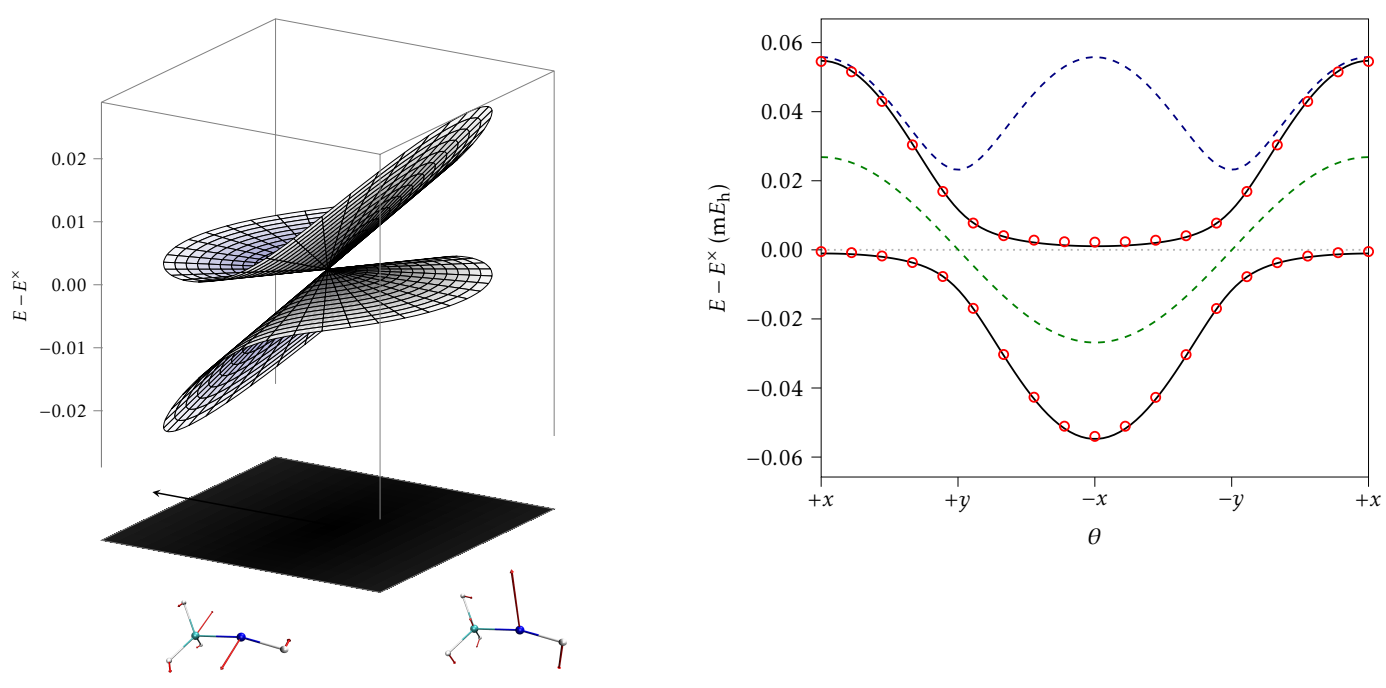

$\begin{array}{rrrr}\text { N } & 0.771060 & -0.000000 & 0.000001 \\ \text { Initial geometry: } & -0.771060 & -0.000000 & -0.000001 \\ \text { H } & -1.156219 & 0.877740 & -0.506761 \\ \text { H } & -1.156221 & -0.000000 & 1.013529 \\ \text { H } & -1.156219 & -0.877740 & -0.506761 \\ \text { H } & 1.156221 & 0.716673 & 0.716673\end{array}$

$\begin{array}{rrrrr} & \text { N } & 0.54846487 & 0.29158547 & 0.15840604 \\ & \mathrm{C} & -0.83417881 & 0.03490953 & 0.00762896 \\ \text { Optimized geometry: } & \mathrm{H} & -1.27084125 & 0.85501167 & -0.55627809 \\ & \mathrm{H} & -1.24692207 & -0.02081754 & 1.01641928 \\ & \mathrm{H} & -0.94723120 & -0.90795729 & -0.52112731 \\ & \mathrm{H} & 1.43827045 & 0.46394116 & 0.61163113\end{array}$

\begin{tabular}{|c|c|c|c|c|}
\hline \multirow{6}{*}{$\hat{x}$ vector: } & $\mathrm{N}$ & -0.177187255 & -0.022335604 & 0.527681257 \\
\hline & C & 0.107916962 & 0.004937929 & -0.750807281 \\
\hline & $\mathrm{H}$ & -0.065625811 & -0.021360231 & 0.104019949 \\
\hline & $\mathrm{H}$ & 0.124383332 & 0.026312699 & 0.181423566 \\
\hline & $\mathrm{H}$ & -0.069602389 & 0.000849130 & 0.103517102 \\
\hline & $\mathrm{H}$ & 0.080115161 & 0.011596077 & -0.165834593 \\
\hline \multirow{6}{*}{$\hat{y}$ vector: } & $\mathrm{N}$ & -0.137499005 & 0.747073957 & -0.014 \\
\hline & C & -0.037660300 & 0.205583060 & -0.004067987 \\
\hline & $\mathrm{H}$ & 0.084655856 & -0.113684021 & -0.15416238 \\
\hline & $\mathrm{H}$ & 0.042157159 & -0.230621260 & 0.00438413 \\
\hline & $\mathrm{H}$ & -0.039649748 & -0.130292022 & 0.15909905 \\
\hline & & 0.087996039 & -0.478059715 & 0.009145310 \\
\hline
\end{tabular}


S6 Methaniminium (f)
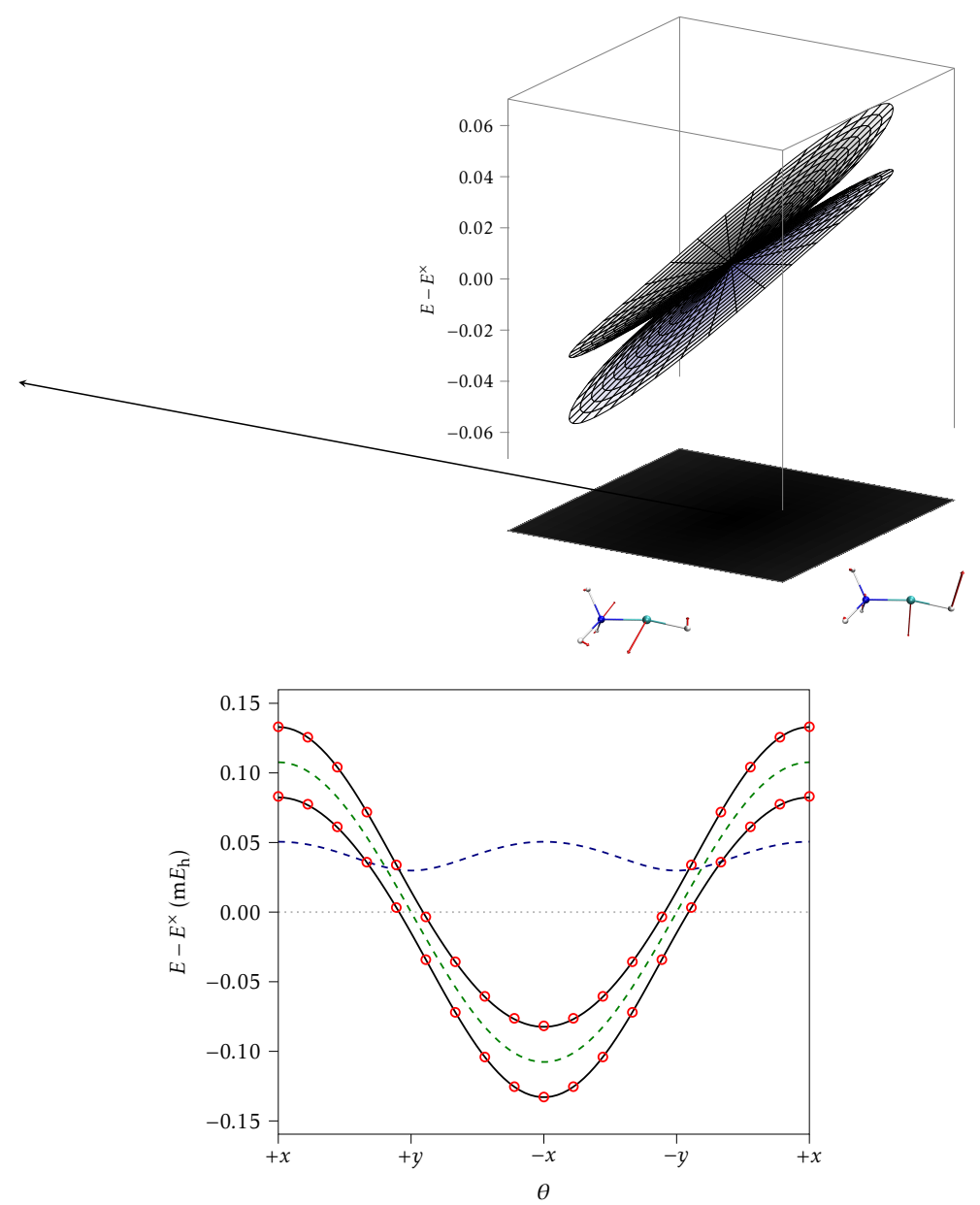

$\begin{array}{rrrr}\text { C } & 0.771060 & -0.000000 & 0.000001 \\ \text { Initial geometry: } & -0.771060 & -0.000000 & -0.000001 \\ \text { H } & -1.156219 & 0.877740 & -0.506761 \\ \text { H } & -1.156221 & -0.000000 & 1.013529 \\ \text { H } & -1.156219 & -0.877740 & -0.506761 \\ \text { H } & 1.156221 & 0.716673 & 0.716673\end{array}$

$\begin{array}{rrrrr} & \text { C } & 0.55489698 & 0.29817515 & 0.15449262 \\ & \text { N } & -0.80845524 & 0.03575459 & -0.01292667 \\ \text { Optimized geometry: } & \text { H } & -1.30235769 & 0.76752628 & -0.51745840 \\ & \text { H } & -1.23951944 & -0.01943800 & 0.91234405 \\ & \text { H } & -0.99601952 & -0.85236781 & -0.47121738 \\ & \text { H } & 1.47901692 & 0.48702279 & 0.65144579\end{array}$

$\hat{x}$ vector: $\begin{array}{rrrr}\mathrm{C} & -0.321845513 & -0.043302993 & 0.621452331 \\ \mathrm{H} & -0.204590359 & 0.021892336 & -0.591297532 \\ \mathrm{H} & 0.132834128 & 0.004494679 & 0.059123130 \\ \mathrm{H} & -0.061280670 & 0.027762056 & 0.095616148 \\ \mathrm{H} & 0.112595636 & 0.014275491 & 0.059944370 \\ & & \end{array}$

$\hat{y}$ vector: $\begin{array}{rrrr}\mathrm{C} & 0.153011987 & -0.807914779 & 0.022819707 \\ \mathrm{H} & -0.022683381 & 0.119063527 & -0.003527423 \\ \mathrm{H} & -0.023528882 & 0.057840037 & 0.129190769 \\ \mathrm{H} & 0.0020803487 & 0.072989152 & -0.002012966 \\ \mathrm{H} & -0.095096634 & 0.055319707 & -0.132319924 \\ & 0.502702355 & -0.014150163\end{array}$ 


\section{S7 Ketene (g)}
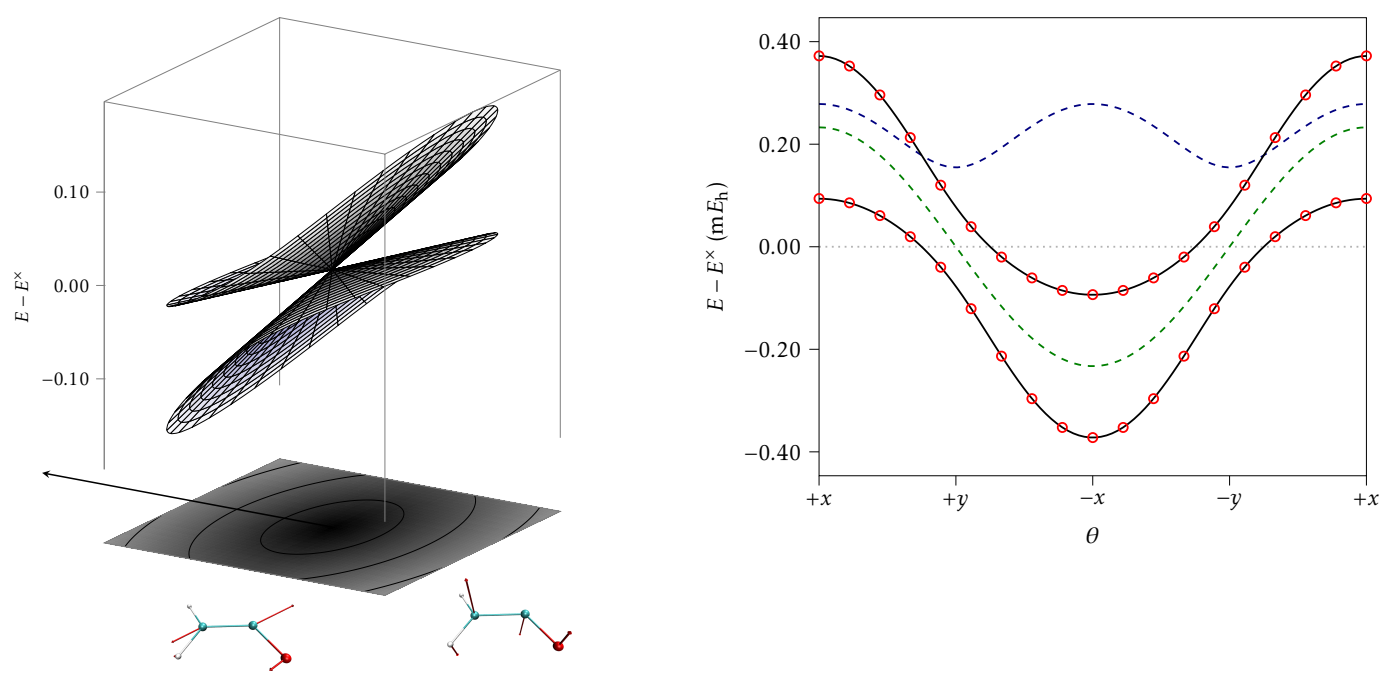

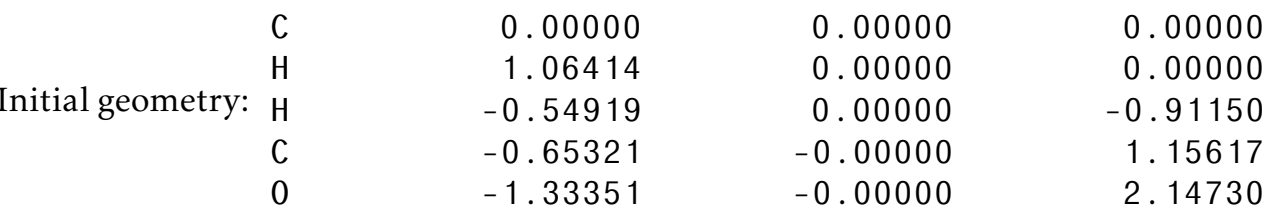

$\begin{array}{rrrrr} & \text { C } & 0.04615061 & -0.00000274 & 0.06335891 \\ \text { Optimized geometry: } & \text { H } & 1.08719124 & 0.00000242 & -0.19655813 \\ & \text { H } & -0.69298507 & 0.00000019 & -0.71844725 \\ \text { C } & -0.37424514 & -0.00000187 & 1.47493754 \\ & 0 & -1.53788164 & 0.00000200 & 1.76867893\end{array}$

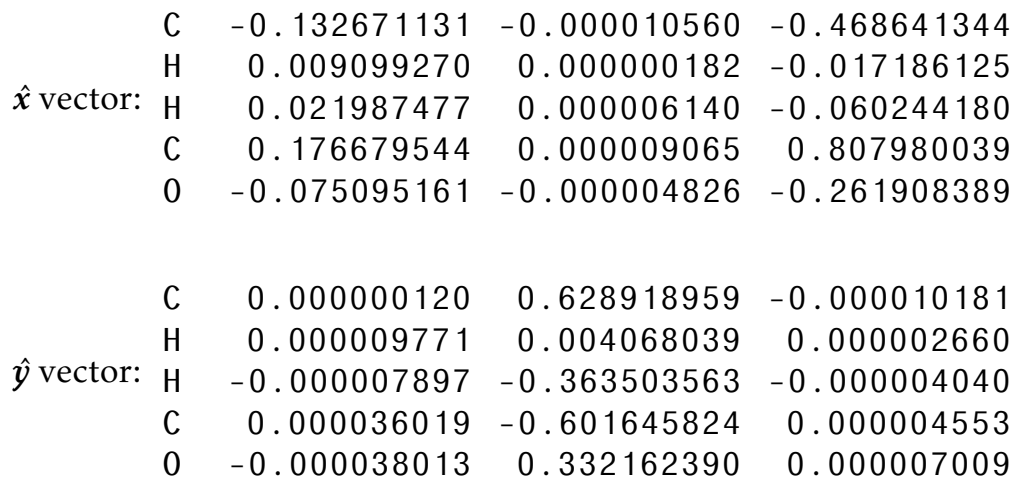


S8 Diazomethane (h)
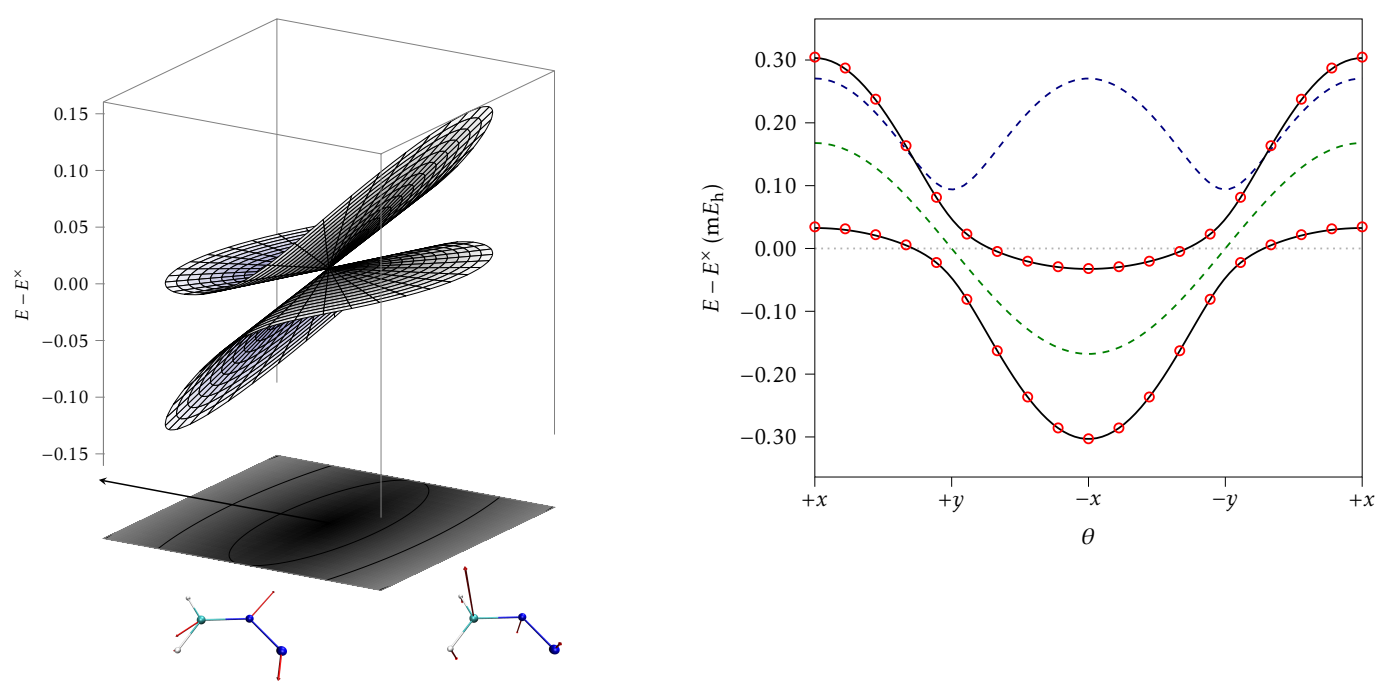

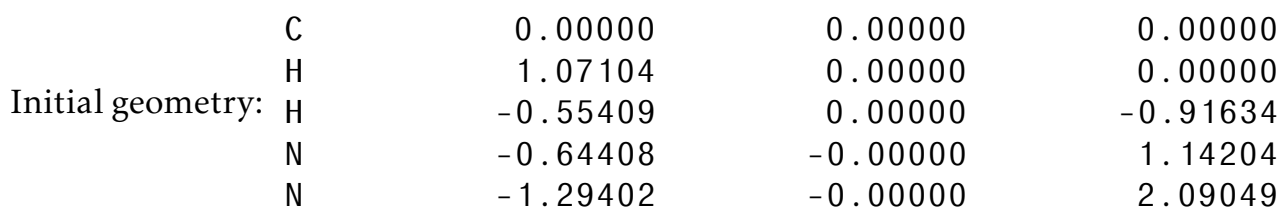

$\begin{array}{rrrrr} & \text { C } & 0.03883422 & 0.00000000 & 0.05444176 \\ \text { Optimized geometry: } & \text { H } & 1.08631051 & 0.00000000 & -0.15849821 \\ & \text { H } & -0.71700571 & -0.00000000 & -0.70552232 \\ \text { N } & -0.33701129 & -0.00000001 & 1.41541787 \\ \text { N } & -1.49227773 & 0.00000001 & 1.71035089\end{array}$

\begin{tabular}{|c|c|c|c|c|}
\hline \multirow{5}{*}{$\hat{x}$ vector: } & C & -0.177053621 & -0.000001579 & -0.385973568 \\
\hline & $\mathrm{H}$ & 0.009676256 & 0.000000403 & -0.018226011 \\
\hline & $\mathrm{H}$ & 0.008766083 & 0.000000762 & -0.05489623 \\
\hline & $\mathrm{N}$ & 0.441883476 & 0.000000872 & 0.69606239 \\
\hline & $\mathrm{N}$ & -0.283272195 & -0.000000458 & -0.23696658 \\
\hline \multirow{5}{*}{$\hat{y}$ vector: } & C & 0.000005337 & 0.781199120 & 0.00000741 \\
\hline & $\mathrm{H}$ & -0.000002732 & -0.153920592 & 0.000001123 \\
\hline & $\mathrm{H}$ & 0.000003259 & -0.361398126 & 0.000002717 \\
\hline & $\mathrm{N}$ & 0.000023532 & -0.449232459 & -0.000016476 \\
\hline & N & -0.000029397 & 0.183352057 & 0.00000522 \\
\hline
\end{tabular}



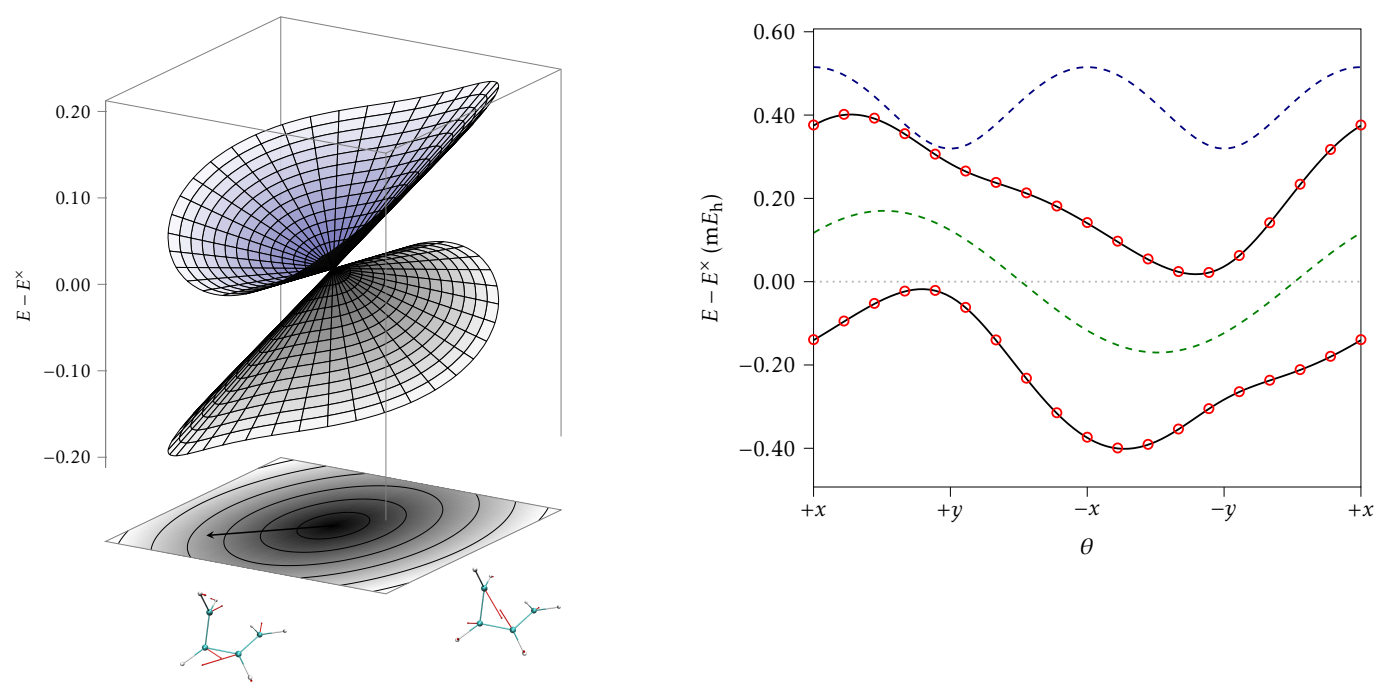

Initial geometry:

$$
0.00000
$$

0.00000

0.00000

$\begin{array}{ll}C & 0.00000 \\ C & 1.51000\end{array}$

0.00000

0.00000

C $\quad 2.20500$

0.00000

1.20378

1.15203

1.52074

$\begin{array}{ll}\mathrm{H} & -0.54050\end{array}$

$-0.63847$

0.68467

$-0.54050$

0.63847

$-0.68467$

0.04900

$-0.93553$

$-0.00000$

1.20378

3.28600

2. 14520

1. 21097

1.00603

2.06358

$\begin{array}{rrr}\text { C } & 0.01296775 \\ \text { C } & 1.46842433 \\ \text { C } & 2.17517041 \\ \text { C } & 1.36540607 \\ \text { Optimized geometry: } & \text { H } & -0.56776206 \\ \text { H } & -0.50082555 \\ \text { H } & 1.98438420 \\ \text { H } & 3.24360126 \\ \text { H } & 1.72176164 \\ \text { H } & 0.48263197\end{array}$

$-0.04013856$

$-0.07602086$

$-0.05381331$

$-0.03300816$

0.00325660

1. 17459663

1. 13422131

1.60179467

$-0.40874244$

0.74592496

0.37693264

$-0.92134982$

0.03851516

$-0.98082222$

0.11494195

1.11874716

2. 13916200

1. 43442049

1. 04792464

2. 20303715

$\begin{array}{rrrr}C & -0.245425852 & 0.148733481 & 0.129850572 \\ \text { C } & 0.680561194 & 0.101776225 & 0.288050103 \\ \text { C } & -0.098175032 & -0.132124171 & -0.380028654 \\ \text { C } & -0.346940942 & 0.002805884 & -0.027525531 \\ \hat{x} \text { vector: } H & 0.005877494 & -0.037445165 & -0.022853034 \\ \text { H } & -0.004067503 & 0.040848321 & 0.019287878 \\ \text { H } & -0.003859617 & -0.107260831 & -0.045053015 \\ \text { H } & -0.001491509 & -0.033261413 & 0.011367109 \\ \text { H } & -0.050214354 & -0.016973949 & -0.097268824 \\ \text { H } & 0.063736121 & 0.032901618 & 0.124173397\end{array}$




\begin{tabular}{|c|c|c|c|c|}
\hline & C & -0.239441349 & -0.061971995 & -0.011175314 \\
\hline & C & -0.010313696 & 0.340962120 & 0.357360563 \\
\hline & C & 0.127663916 & -0.154217172 & 0.334706389 \\
\hline & C & 0.142523740 & -0.331181476 & -0.621222182 \\
\hline$\hat{v}$ vector: & $\mathrm{H}$ & 0.004962010 & -0.016748337 & -0.003090943 \\
\hline & $\mathrm{H}$ & 0.006212182 & 0.034171528 & 0.023275323 \\
\hline & $\mathrm{H}$ & 0.013780431 & 0.099784782 & 0.016301119 \\
\hline & $\mathrm{H}$ & -0.016849344 & 0.061765492 & -0.040901728 \\
\hline & $\mathrm{H}$ & 0.006339189 & 0.025896639 & 0.021923991 \\
\hline & $\mathrm{H}$ & -0.034877079 & 0.001538418 & -0.077177219 \\
\hline
\end{tabular}




\section{S10 Butadiene $(\mathbf{j})$}
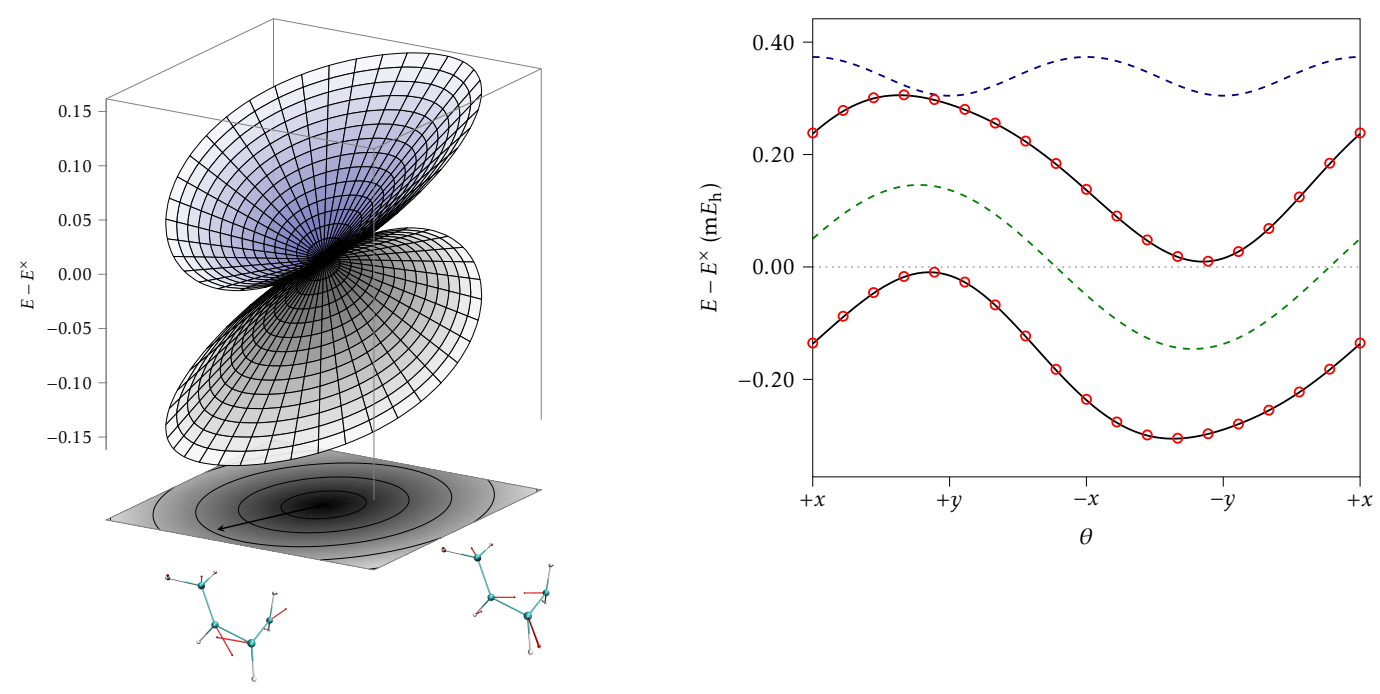

(2)

C

0.00000

C $\quad 1.48000$

C $\quad 1.48000$

C $\quad 1.34602$

Initial geometry: $\mathrm{H} \quad-0.54050$

$-0.54050$

2.03027

1.94809

0.40547

2. 18873

C

0.03135587

C $\quad 1.49353330$

C $\quad 1.61359309$

C $\quad 1.32201770$

Optimized geometry:

$-0.58244910$

$-0.44250078$

1.94214137

2. 01031800

0.32007071

2. 08949985
0.00000

0.00000

0.00000

1.27470

$-0.93603$

0.93603

$-0.75738$

$-0.81075$

1.80748

1.67296

0.02547676

$-0.09049104$

0.13306075

1. 34177725

$-0.84968996$

0.98011500

$-0.98636882$

$-0.68846527$

1. 65688527

1.66471007
0.00000

0.00000

1.47000

2. 21000

$-0.01634$

0.01634

$-0.54050$

2. 01050

2. 75754

$-0.09225431$

$-0.05478053$

1. 34957194

2. 14789800

0.03428510

0.04264472

$-0.45001619$

1. 93153076

2. 37053190

2. 83108860
2. 20296 


$\begin{array}{rrrr}\text { C } & 0.128748884 & -0.089644036 & 0.430338959 \\ \text { C } & 0.486661009 & 0.116537583 & -0.381976749 \\ \text { C } & -0.434704146 & -0.143903707 & -0.244246811 \\ \text { C } & 0.023854266 & 0.177626509 & 0.105190236 \\ \text { vector: } & -0.012189430 & 0.006079923 & 0.097897606 \\ \text { H } & -0.011797405 & 0.010244558 & -0.057835219 \\ \text { H } & -0.005187249 & 0.006261691 & -0.000238374 \\ \text { H } & -0.211433890 & -0.060857032 & 0.024445811 \\ \text { H } & 0.008401237 & -0.075663397 & 0.080925765 \\ \text { H } & 0.027646724 & 0.053317908 & -0.054501224\end{array}$




\section{S11 Butadiene (k)}
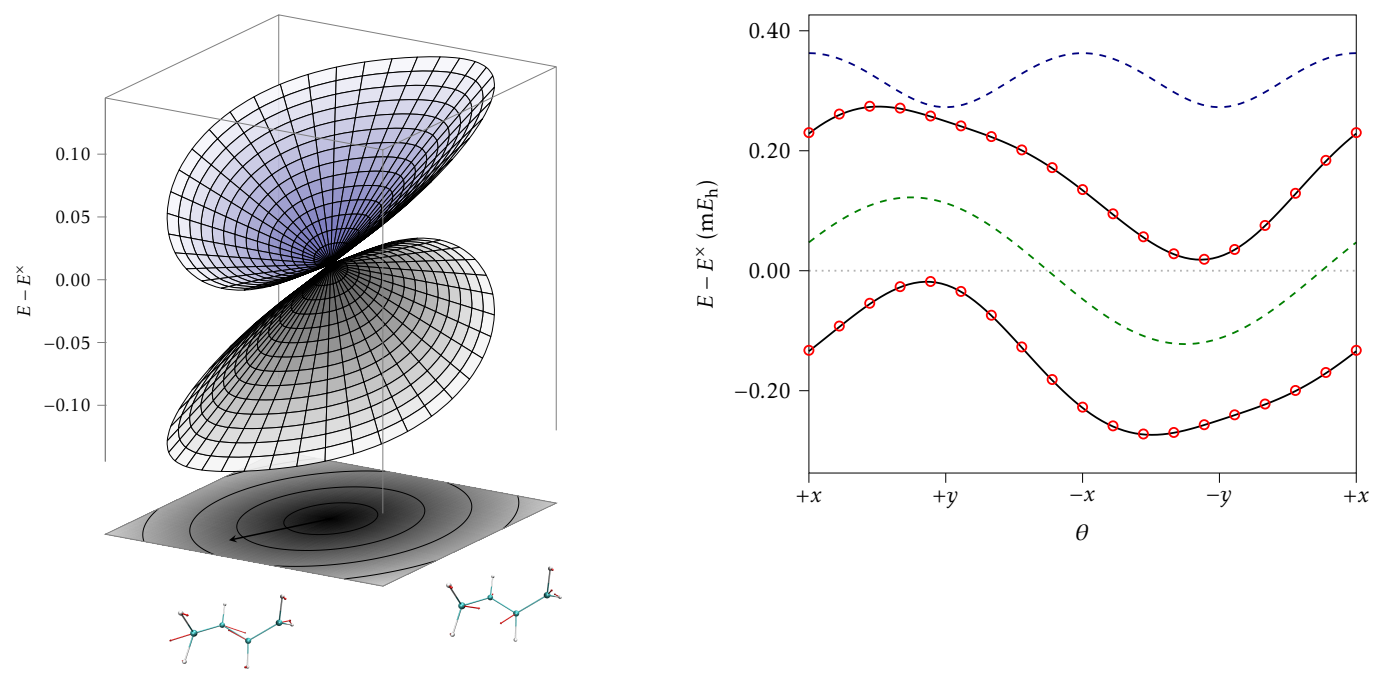

Initial geometry:

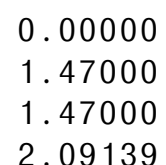

0.00000

0.00000

C

0.00000

0.00000

C $\quad 1.47000$

0.00000

1.41000

1.12102

2. 15000

0.93617

0.00000

$-0.54050$

$-0.93617$

0.00000

0.81880

$-0.54050$

1.07436

$-0.84846$

1.95050

1.57156

2.06474

2. 23787

3.06508

0.99609

2.60263

$\begin{array}{rrr}\text { C } & -0.03883376 \\ \text { C } & 1.41230374 \\ \text { C } & 1.50068959 \\ \text { C } & 2.05179916 \\ \text { Optimized geometry: } & \text { H } & -0.66088880 \\ \text { H } & -0.52398514 \\ \text { H } & 1.94298054 \\ \text { H } & 1.12890684 \\ \text { H } & 1.73041600 \\ \text { H } & 3.04187183\end{array}$

$-0.00363117$

$-0.12604806$

0.11438335

0.03864595

$-0.02046781$

1. 45296084

1.07176945

2. 25015866

0.87694426

$-0.11218414$

$-0.93163219$

0.12467736

0.91526034

$-0.44774797$

$-0.91459355$

1. 91873484

2. 07751951

2. 04308953

0.96663782

2. 66821298

$\begin{array}{rrrr}\text { C } & -0.309890730 & -0.201738896 & -0.256366490 \\ \text { C } & 0.309669851 & -0.052918747 & 0.504137065 \\ \text { C } & -0.086579508 & 0.050458361 & -0.501913042 \\ \text { C } & 0.234739488 & 0.087118727 & 0.170371478 \\ H & 0.038388813 & 0.006444320 & 0.139433162 \\ \text { H } & -0.014035586 & -0.003656557 & -0.060197337 \\ \text { H } & 0.005311584 & 0.001684926 & 0.001856146 \\ \text { H } & -0.164909467 & 0.088003036 & 0.000261092 \\ \text { H } & -0.073579506 & -0.027200486 & 0.097917843 \\ \text { H } & 0.060885062 & 0.051805316 & -0.095499918\end{array}$




$\begin{array}{rrrr}C & 0.045350504 & 0.128586843 & 0.395679790 \\ \text { C } & 0.576693311 & -0.159316645 & -0.333119745 \\ \text { C } & -0.484271666 & -0.095275700 & -0.066994001 \\ \text { C } & -0.122721391 & 0.163781425 & 0.067172674 \\ \text { vector: } H & -0.029667096 & 0.003472258 & 0.122866217 \\ \text { H } & -0.009049671 & -0.020958974 & -0.070127872 \\ \text { H } & 0.011555983 & -0.019746140 & -0.010483080 \\ \text { H } & -0.015934762 & 0.009832155 & -0.018448284 \\ \text { H } & -0.041789892 & -0.014739050 & 0.075222838 \\ \text { H } & 0.069834680 & 0.004363829 & -0.161768536\end{array}$




\section{S12 Benzene (1)}
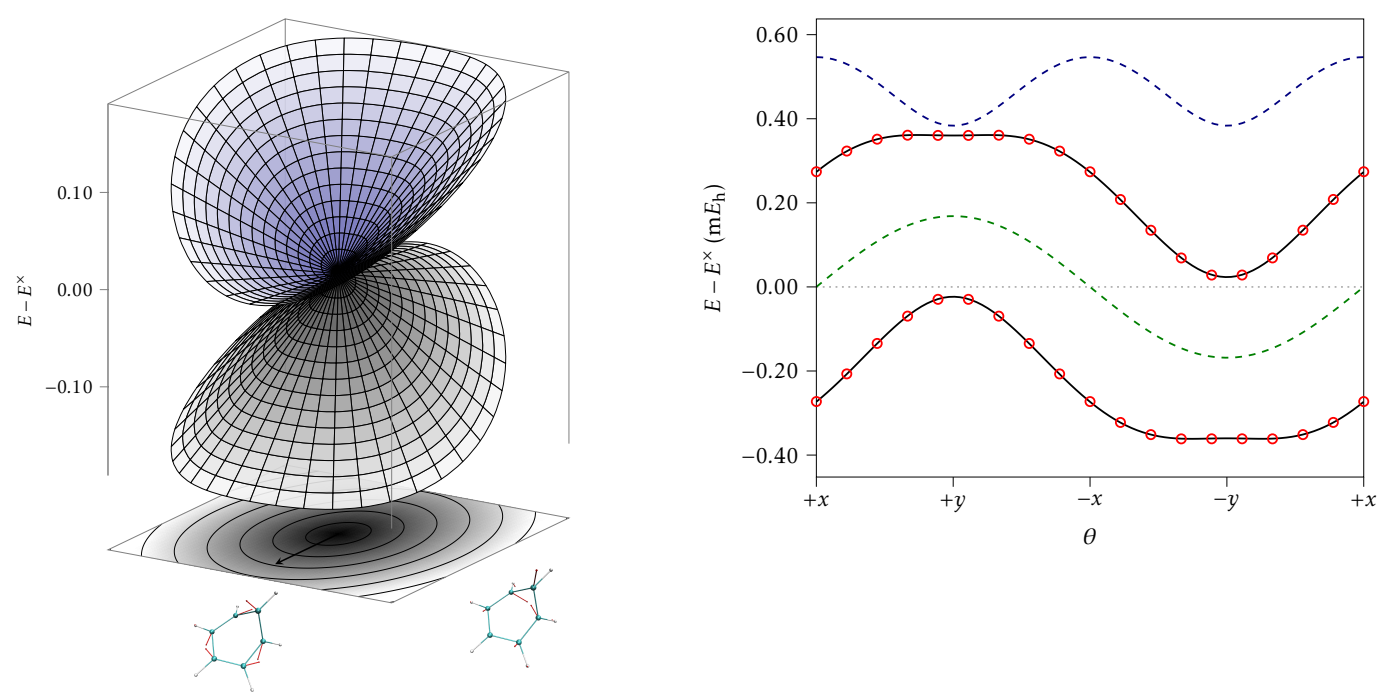

Initial geometry: $\begin{gathered}\mathrm{C} \\ \mathrm{C} \\ \mathrm{C} \\ \mathrm{C} \\ \mathrm{C} \\ \mathrm{H} \\ \mathrm{H} \\ \mathrm{H} \\ \mathrm{H} \\ \mathrm{H} \\ \mathrm{H}\end{gathered}$

0.00000

1.39000

2.03441

1.46393

0.38195

C

c

C

H

$-0.53215$

$-0.53500$

1.98517

2. 99189

2. 03615

0.15152

$-1.58992$

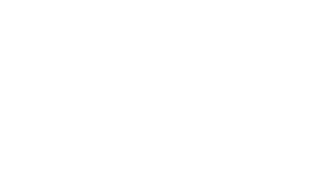

Optimized geometry:

$\begin{array}{ll}\mathrm{C} & 0.01335433 \\ \mathrm{C} & 1.40527700 \\ \mathrm{C} & 2.01519497 \\ \mathrm{C} & 1.48924722 \\ \mathrm{C} & 0.38697309\end{array}$

C $\quad-0.55396961$

H $\quad-0.56225379$

$\mathrm{H} \quad 2.01465469$

2. 91192386

2. 05303676

0.20620362

$-1.60169214$
0.006936342

0.009101950

0.093941010

0.007477502

$-0.102443827$

C $\quad-0.324319925$

C $\quad 0.392393819$

H -0.006259928

H -0.008511761

H -0.008467184

H $\quad-0.019004098$

H -0.013276459

H -0.005091055
0.000141229

0.059085158

$-0.027602995$

0.000434883

0.028127547
$-0.016469502$

$-0.058729297$
0.00000

0.00000

0.00000

$-0.93565$

0.04158

0.04837

0.00000

$-0.08076$

0.44075

$-1.24240$

0.51223

0.00920

$-0.05335974$

$-0.02633136$

$-0.09375997$

$-1.00096017$

$-0.05574127$

0.01967621

$-0.06644067$

0.09124473

0.46813004

$-1.19640868$

0.53125306

0.17601782

0.187612503

0.185270908

$-0.449389592$

0.205953624

0.216561866

$-0.384439846$

0.003968635

$-0.000539345$

0.011715881

0.012051150

0.002068911

0.009165304
$-0.04143839$

$-0.01574308$

1. 31377374

2. 31870126

2. 34728228

1. 22799422

$-0.94736546$

$-0.89203952$

1. 51464892

3. 21403055

3. 23212656

1. 40368891 


\begin{tabular}{|c|c|c|c|c|}
\hline & C & -0.025551565 & 0.067887883 & -0.042418911 \\
\hline & C & -0.075620454 & -0.036881637 & -0.133815784 \\
\hline & C & -0.253496056 & 0.261583013 & 0.390744755 \\
\hline & C & 0.007378232 & -0.573451959 & 0.032516164 \\
\hline & C & 0.469657449 & 0.244662181 & -0.068474066 \\
\hline & C & -0.089900832 & -0.036574615 & -0.124694487 \\
\hline \multirow{6}{*}{$\hat{y}$ vector: } & $\mathrm{H}$ & 0.000969008 & 0.001653714 & 0.001451806 \\
\hline & $\mathrm{H}$ & -0.005113155 & -0.054148434 & -0.017022430 \\
\hline & $\mathrm{H}$ & -0.0866 & 0.092707131 & 0.0 \\
\hline & $\mathrm{H}$ & -0.005651936 & -0.002381519 & -0.008813517 \\
\hline & $\mathrm{H}$ & 0.078968082 & 0.088828122 & -0.061909041 \\
\hline & & -0.015030732 & -0.053883879 & -0.010737893 \\
\hline
\end{tabular}




\section{S13 Fulvene (m)}

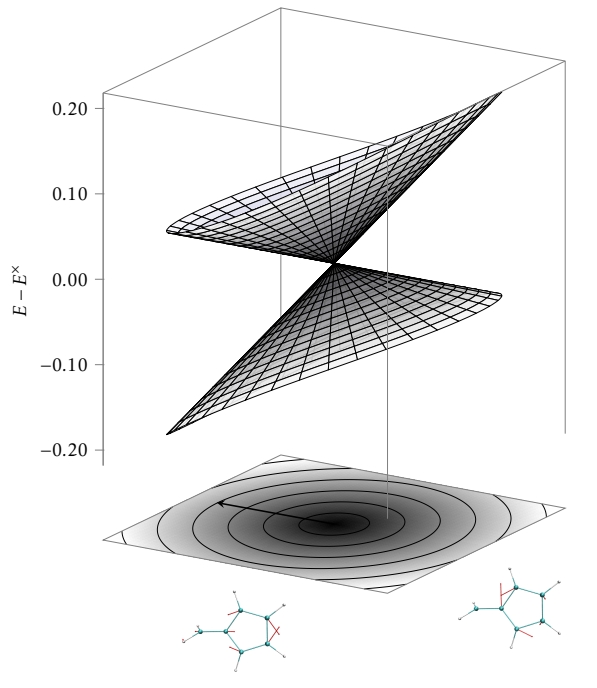

$\begin{array}{rrrr}\text { C } & -0.440320 & 1.280980 & 0.000000 \\ \text { C } & 0.747610 & 2.174310 & 0.000000 \\ \text { C } & 1.915590 & 1.281710 & 0.000000 \\ \text { C } & 1.476000 & -0.000000 & 0.000000 \\ \text { Initial geometry: } & -0.000000 & 0.000000 & 0.000000 \\ \text { C } & 0.745930 & 3.523310 & 0.000000 \\ \text { H } & 1.527228 & 4.117829 & -0.451510 \\ \text { H } & -0.144121 & 4.115657 & 0.156810 \\ \text { H } & -0.574280 & -0.902200 & 0.000000 \\ \text { H } & 2.921670 & 1.645820 & 0.0000000 \\ \text { H } & -1.445730 & 1.646480 & 0.000000\end{array}$

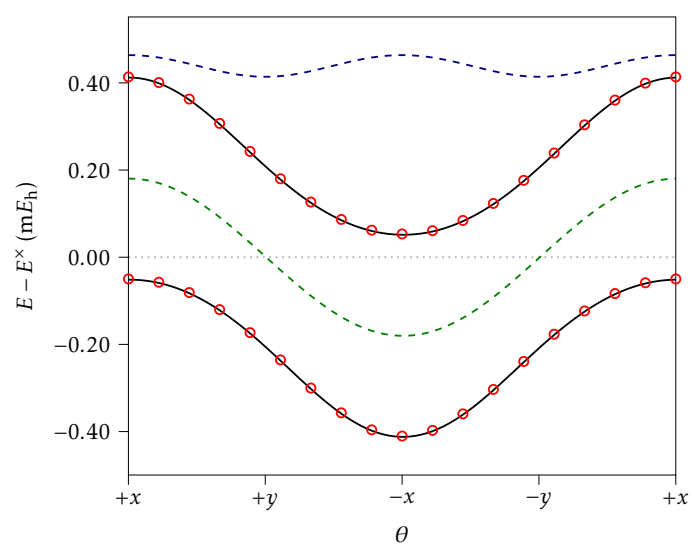

\begin{tabular}{|c|c|c|c|c|c|}
\hline & C & -0.38534268 & 1.30897569 \\
\hline & & & C & 0.74111310 & 2. 14610593 \\
\hline & & & C & 1.86582496 & 1.31015751 \\
\hline & & & C & 1.42099432 & -0.07760645 \\
\hline & & & C & 0.05736593 & -0.07819655 \\
\hline \multirow{7}{*}{\multicolumn{3}{|c|}{ Optimized geometry: }} & C & 0.74480095 & 3.62318177 \\
\hline & & & $\mathrm{H}$ & 1.16131887 & 4. 15908357 \\
\hline & & & H & 0.21622089 & 4.18707640 \\
\hline & & & $\mathrm{H}$ & 2.06322955 & -0.93318732 \\
\hline & & & $\mathrm{H}$ & -0.58619659 & -0.93437302 \\
\hline & & & $\mathrm{H}$ & 2.88474407 & 1.63657590 \\
\hline & & & $\mathrm{H}$ & -1.40414638 & 1.63391257 \\
\hline \multirow{12}{*}{$\hat{x}$ vector: } & C & \multicolumn{2}{|c|}{0.130014380} & 0.350729991 & -0.078964637 \\
\hline & C & \multicolumn{2}{|c|}{-0.007713303} & -0.256915928 & 0.005088185 \\
\hline & C & \multicolumn{2}{|c|}{-0.125598200} & 0.348294867 & 0.063881478 \\
\hline & C & \multicolumn{2}{|c|}{-0.450762366} & -0.309810792 & -0.032658066 \\
\hline & C & \multicolumn{2}{|c|}{0.451385694} & -0.310363961 & 0.042807055 \\
\hline & $\mathrm{C}$ & & 0.171596724 & -0.001169928 \\
\hline & $\mathrm{H}$ & \multicolumn{2}{|c|}{-0.079959346} & -0.000491688 & -0.039354270 \\
\hline & $\mathrm{H}$ & \multicolumn{2}{|c|}{0.070508315} & 0.006448122 & 0.039573807 \\
\hline & $\mathrm{H}$ & \multicolumn{2}{|c|}{-0.002768191} & -0.002528115 & -0.011005748 \\
\hline & $\mathrm{H}$ & \multicolumn{2}{|c|}{0.002829386} & -0.002288698 & 0.011825171 \\
\hline & $\mathrm{H}$ & \multirow{2}{*}{\multicolumn{2}{|c|}{0.002130210}} & 0.002731720 & 0.003306638 \\
\hline & $\mathrm{H}$ & & & 0.002597759 & -0.003329684 \\
\hline
\end{tabular}




\begin{tabular}{|c|c|c|c|c|}
\hline & C & -0.211654817 & -0.433462860 & -0.008888276 \\
\hline & C & 0.689833270 & -0.001892784 & 0.089974814 \\
\hline & C & -0.214092096 & 0.439483542 & -0.019518737 \\
\hline & C & -0.121870024 & -0.083766989 & -0.021344626 \\
\hline & C & -0.115171581 & 0.077934007 & -0.025036943 \\
\hline & C & -0.018880066 & 0.000388133 & -0.032345242 \\
\hline & $\mathrm{H}$ & -0.001949446 & -0.000703492 & 92904 \\
\hline & $\mathrm{H}$ & 0.000919810 & 0.001935505 & 229239 \\
\hline & $\mathrm{H}$ & -0.002096892 & -0.004790302 & 0.005822761 \\
\hline & $\mathrm{H}$ & -0.002067439 & 0.004929816 & 0.006554133 \\
\hline & $\mathrm{H}$ & -0.001419785 & 0.002210459 & -0.002413408 \\
\hline & H & -0.001550934 & -0.002265035 & -0.002026619 \\
\hline
\end{tabular}



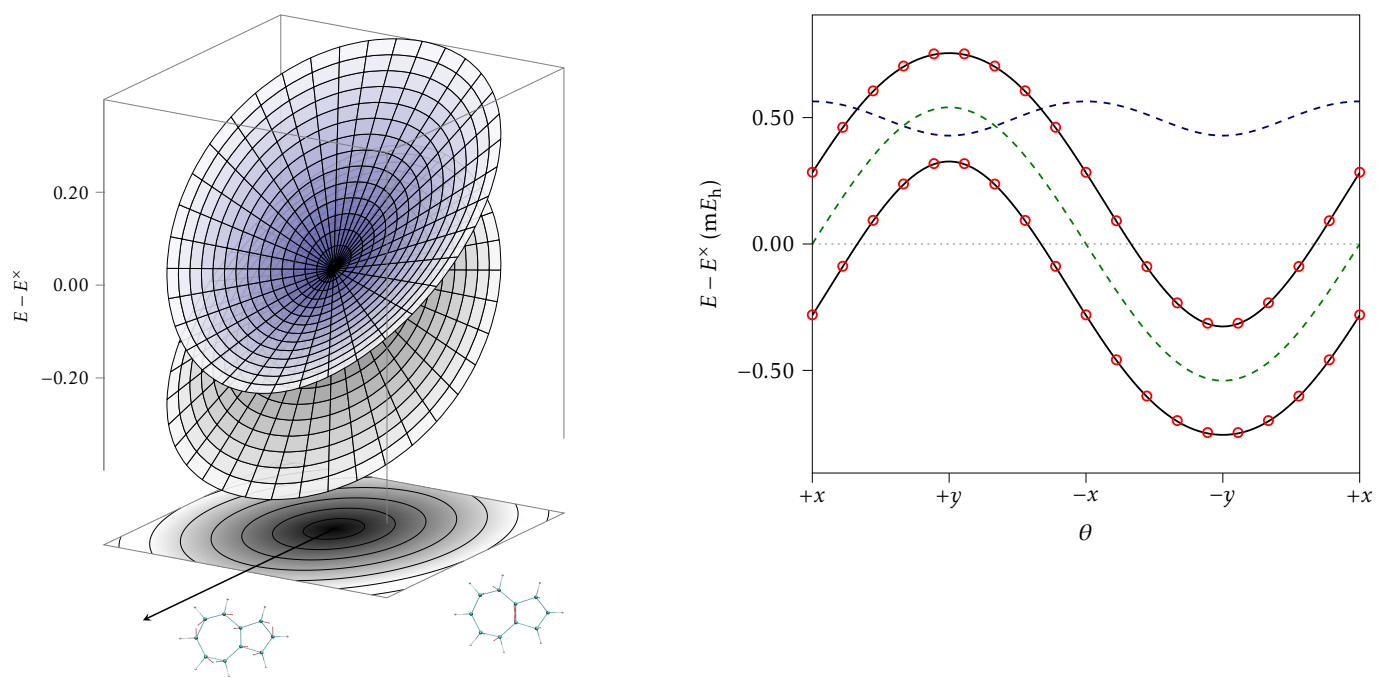

Initial geometry: $\mathrm{C}$

$$
\begin{array}{r}
0.00000 \\
1.40762 \\
1.88593 \\
0.77357 \\
0.85491 \\
-0.20203 \\
-1.57527 \\
-2.27474
\end{array}
$$

$-1.77813$

$-0.44609$

$-0.63346$

2. 03205

2. 92595

1. 87440

0.08664

$-2.21167$

$-3.37517$

$-2.52787$

$\begin{array}{rrr}\text { C } & 0.08126250 \\ \text { C } & 1.47047604 \\ \text { C } & 1.89803685 \\ \text { C } & 0.68730831 \\ \text { C } & 0.83439903 \\ \text { C } & -0.19421396 \\ \text { C } & -1.61563896 \\ \text { C } & -2.31342325 \\ \text { Optimized geometry: } & -1.83387572 \\ \text { C } & -0.37900829 \\ \text { H } & -0.58516530 \\ \text { H } & 2.08489233 \\ \text { H } & 2.91646836 \\ \text { H } & 1.83784020 \\ \text { H } & 0.08483413 \\ \text { H } & -2.23247947 \\ \text { H } & -3.38811669 \\ \text { H } & -2.53695611\end{array}$
0.00000
0.00000
0.00000
0.00000
0.00000
1. 32374
0.00000
2. 19984
0.00000
3. 58714
0.00000
4. 49843
0.00000
4. 25146
0.00000
3.04414
0.00000
1. 74010
0.00000
1.34369
$-0.00000$
$-0.87699$
$-0.00000$
$-0.88972$
1. 62162
4. 01514
5. 56421
5. 15844
3.13563
0.92741

\subsection{0 \\ 0.00000000}

$-0.04192444$

$-0.00000000$

$-0.08997443$

0.00000000

1. 23271424

0.00000000

2. 16031163

0.00000000

$-0.00000000$

$-0.00000000$

0.00000000

0.00000000

$-0.00000000$

0.00000000

$-0.00000000$

0.00000000

0.00000000

$-0.00000000$

3.65426773

4.53643695

4. 30873593

3. 04959531

1. 78220594

1. 41217845

$-0.88382672$

$-0.96571386$

1. 57289359

4. 03887769

5. 57835167

5. 18793060

$-0.00000000$

0.00000000

.14172472

0.96949501 


$\begin{array}{rrrr}\text { C } & 0.245435050 & -0.000000000 & -0.215084119 \\ \text { C } & -0.292101447 & 0.000000000 & -0.204926612 \\ \text { C } & -0.118721253 & 0.000000000 & 0.303960806 \\ \text { C } & 0.159146427 & -0.000000000 & -0.195913659 \\ \text { C } & -0.154421024 & -0.000000000 & 0.268410362 \\ \text { C } & 0.329309104 & 0.000000000 & -0.083262048 \\ \text { C } & -0.271082582 & -0.000000000 & -0.190173247 \\ \text { C } & 0.033756201 & -0.000000000 & 0.337955284 \\ \text { C } & 0.199811309 & 0.000000000 & -0.236504723 \\ \text { C } & -0.129995708 & -0.000000000 & 0.216337123 \\ \text { H } & 0.000339807 & 0.000000000 & 0.001966929 \\ \text { H } & -0.004420188 & -0.000000000 & -0.003102900 \\ \text { H } & 0.001964147 & -0.000000000 & -0.000354555 \\ \text { H } & -0.001158968 & 0.000000000 & 0.004752313 \\ \text { H } & 0.003084037 & 0.000000000 & -0.000291938 \\ \text { H } & -0.005783341 & -0.000000000 & -0.004055376 \\ \text { H } & 0.000771735 & -0.000000000 & 0.002999022 \\ \text { H } & 0.004066694 & 0.000000000 & -0.002712661 \\ & & & \\ \text { C } & 0.117385179 & -0.000000000 & -0.107420287 \\ \text { C } & 0.032190167 & 0.000000000 & -0.045899107 \\ \text { C } & 0.061098719 & -0.000000000 & -0.146936448 \\ \text { C } & -0.533518281 & 0.000000000 & -0.304080226 \\ \text { C } & -0.019128367 & -0.000000000 & 0.257967250 \\ \text { C } & 0.134752786 & -0.000000000 & -0.078872744 \\ \text { C } & -0.055151259 & -0.000000000 & 0.078670118 \\ \text { C } & 0.028307706 & 0.000000000 & -0.153616753 \\ \text { C } & -0.236091789 & -0.000000000 & 0.105808439 \\ \text { C } & 0.467505279 & 0.000000000 & 0.398163211 \\ \text { H } & 0.003273437 & -0.000000000 & -0.004859587 \\ \text { H } & -0.001290299 & -0.000000000 & 0.001840915 \\ \text { H } & 0.003456495 & -0.000000000 & -0.004731212 \\ \text { H } & -0.000559438 & 0.000000000 & 0.005416694 \\ \text { H } & 0.003327471 & 0.000000000 & 0.000176348 \\ \text { H } & 0.000649937 & -0.000000000 & -0.000926417 \\ \text { H } & -0.001299139 & 0.000000000 & -0.003068988 \\ \text { H } & -0.004908603 & 0.000000000 & 0.002368794\end{array}$


S15 s-Indacene (o)
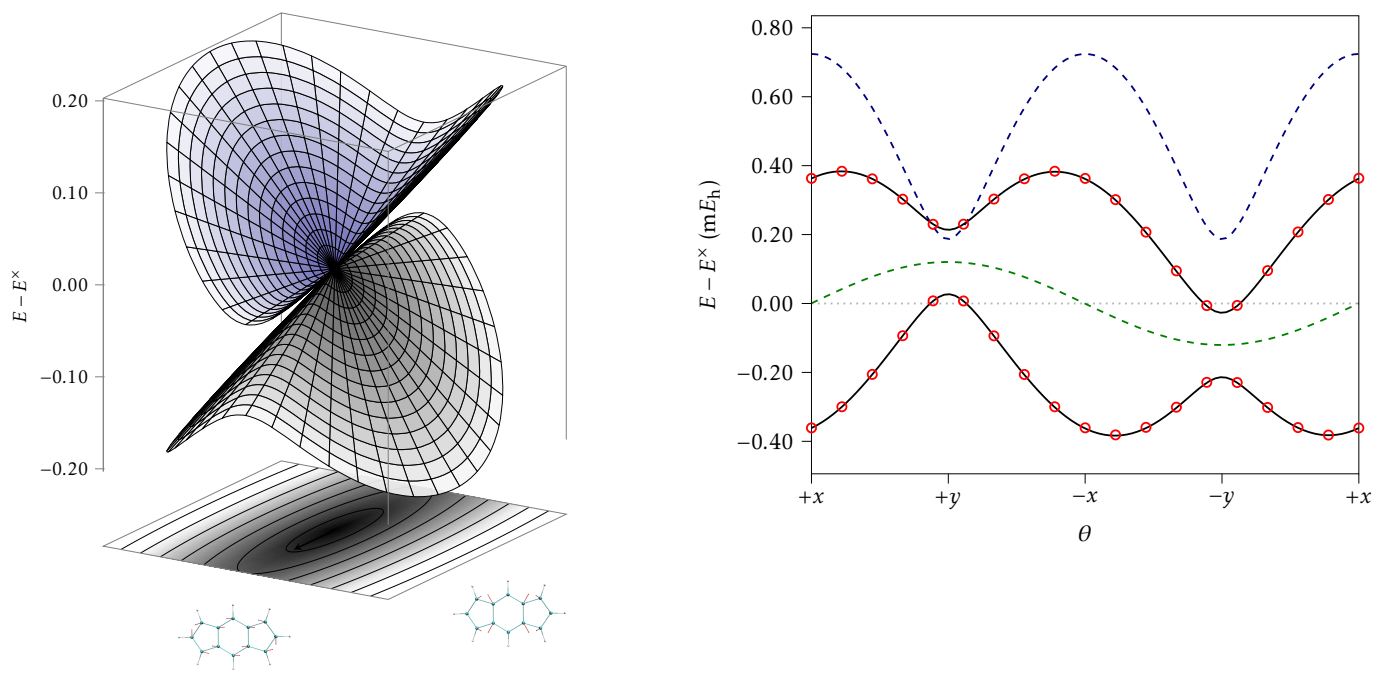

Initial geometry: $\begin{array}{r}\mathrm{C} \\ \mathrm{C} \\ \mathrm{C} \\ \mathrm{C} \\ \mathrm{C} \\ \mathrm{C} \\ \mathrm{C} \\ \mathrm{C} \\ \mathrm{C} \\ \mathrm{C} \\ \mathrm{H} \\ \mathrm{H} \\ \mathrm{H} \\ \mathrm{H} \\ \mathrm{H} \\ \mathrm{H} \\ \mathrm{H} \\ \mathrm{H}\end{array}$

\begin{abstract}
0.00000
1. 36712

2. 15830

1. 52117

0.15405

$-0.63724$

2. 32723

3. 58789

3. 49701
\end{abstract}

$-1.97588$

$-2.06662$

$-0.80588$

$-0.59802$

2. 11916

2. 05273

4. 52724

4. 35487

$-2.83380$

$-3.00593$

$-0.53127$

Optimized geometry:

C
$-0.02693726$
1. 37081688
2. 17687275
1. 54815040
0.15039590
$-0.65565970$
2. 28315968
3. 58932504
3.52574752

$-2.00453448$

$-2.06811221$

$-0.76194645$

$-0.60177722$

2. 12299017

2. 03982344

4. 49609910

4. 36450052

$-2.84328726$

$-2.97488636$

$-0.51861044$
0.00000

0.00000

0.00000

0.00000

0.00000

0.00000

$-0.00000$

0.00000

0.00000

0.00000

0.00000

$-0.00000$

$-0.00000$

$-0.00000$

$-0.00000$

0.00000

0.00000

0.00000

0.00000

$-0.00000$

0.00000000

0.00000000

0.00000000

0.00000000

0.00000000

0.00000000

$-0.00000000$

$-0.00000000$

0.00000000

0.00000000

$-0.00000000$

0.00000000

0.00000000

0.00000000

$-0.00000000$

$-0.00000000$

$-0.00000000$

$-0.00000000$

$-0.00000000$

$-0.00000000$
0.00000

0.00000

1.24146

2. 52474

2. 52455

1. 28321

$-1.10921$

$-0.56983$

0.88407

1. 64077

3.09465

3.63392

$-0.91732$

3. 44205

$-2.15684$

$-1.11034$

1.54927

0.97567

3.63525

4.68152

0.01765878

$-0.01569885$

1. 25826247

2. 50710039

2. 54045834

1. 26649704

$-1.07597061$

$-0.52744715$

0.88782434

1. 63693443

3. 05220584

3. 60072965

$-0.89084230$

3. 41560161

$-2.11937037$

$-1.10114991$

1. 55445179

0.97030667

3. 62590841

4. 64412942 


$\begin{array}{rrrr}\text { C } & -0.230970658 & -0.000000000 & 0.146135778 \\ \text { C } & 0.233857983 & -0.000000000 & -0.126648472 \\ \text { C } & -0.214551142 & 0.000000000 & 0.157231501 \\ \text { C } & 0.230970939 & -0.000000000 & -0.146136075 \\ \text { C } & -0.233858357 & -0.000000000 & 0.126648654 \\ \text { C } & 0.214551100 & 0.000000000 & -0.157231063 \\ \text { C } & -0.288888856 & 0.000000000 & 0.082661887 \\ \text { C } & 0.171748281 & 0.000000000 & 0.271466343 \\ \text { C } & 0.198432383 & -0.000000000 & -0.225683226 \\ \hat{x} \text { vector: C } & -0.198432581 & -0.000000000 & 0.225683272 \\ \text { C } & -0.171748172 & 0.000000000 & -0.271466652 \\ \text { C } & 0.288889059 & 0.000000000 & -0.082661953 \\ \text { H } & -0.001534492 & 0.000000000 & 0.000967415 \\ \text { H } & 0.001534497 & 0.000000000 & -0.000967415 \\ \text { H } & -0.002681561 & -0.000000000 & -0.001062708 \\ \text { H } & 0.001038959 & 0.000000000 & 0.001639068 \\ \text { H } & 0.000190579 & 0.000000000 & -0.002879705 \\ \text { H } & -0.000190578 & -0.000000000 & 0.002879696 \\ \text { H } & -0.001038953 & 0.000000000 & -0.001639060 \\ \text { H } & 0.002681570 & -0.000000000 & 0.001062715 \\ & & & \\ \text { C } & -0.042982937 & 0.000000000 & -0.068000372 \\ \text { C } & -0.021622210 & -0.000000000 & -0.446697171 \\ \text { C } & 0.394202626 & -0.000000000 & 0.210849648 \\ \text { C } & 0.042985741 & 0.000000000 & 0.067998874 \\ \text { C } & 0.021618200 & -0.000000000 & 0.446699862 \\ \text { C } & -0.394204534 & -0.000000000 & -0.210848385 \\ \text { C } & -0.214451470 & 0.000000000 & 0.002041431 \\ \text { C } & -0.031408735 & -0.000000000 & 0.019792429 \\ \text { C } & -0.093759726 & 0.000000000 & 0.193100153 \\ \text { C vector } & 0.093760226 & 0.000000000 & -0.193100281 \\ \text { C } & 0.031408109 & -0.000000000 & -0.019793368 \\ \text { C } & 0.214454633 & 0.000000000 & -0.002042610 \\ \text { H } & 0.005125877 & 0.000000000 & 0.008123816 \\ \text { H } & -0.005126035 & -0.000000000 & -0.008123884 \\ \text { H } & -0.003625242 & -0.000000000 & 0.006489928 \\ \text { H } & 0.003140525 & -0.000000000 & -0.001981413 \\ \text { H } & -0.007409546 & -0.000000000 & 0.000499940 \\ \text { H } & 0.007409464 & -0.000000000 & -0.000499791 \\ \text { H } & -0.003140342 & -0.000000000 & 0.001981254 \\ \text { H } & 0.003625374 & -0.000000000 & -0.006490061\end{array}$



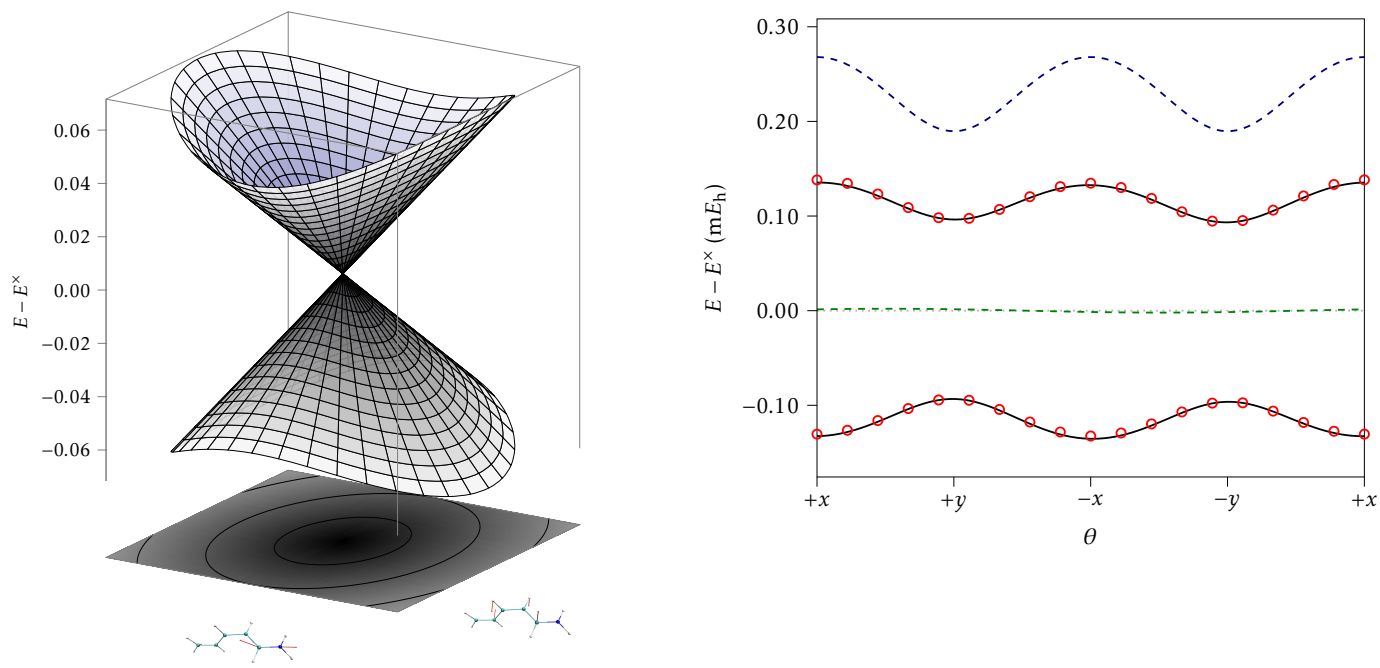

\begin{tabular}{|c|c|c|c|c|}
\hline $\mathrm{C}$ & & 0.00000 & 0.00000 & 0.00000 \\
\hline C & & 1.35449 & 0.00000 & 0.00000 \\
\hline $\mathrm{C}$ & & 2.05966 & 0.00000 & 1.25446 \\
\hline C & & 3.42859 & 0.00016 & 1.39722 \\
\hline C & & 4.18603 & -1.20925 & 1.45488 \\
\hline $\mathrm{N}$ & & 5.49261 & -1.16618 & 1.59211 \\
\hline Initial geometry: $\mathrm{H}$ & & -0.56266 & -0.00014 & -0.92583 \\
\hline $\mathrm{H}$ & & -0.57784 & 0.00028 & 0.91922 \\
\hline $\mathrm{H}$ & & 1.90671 & -0.00024 & -0.94447 \\
\hline $\mathrm{H}$ & & 1.44779 & 0.00033 & 2. 17176 \\
\hline $\mathrm{H}$ & & 3.96261 & 0.94837 & 1.46996 \\
\hline $\mathrm{H}$ & & 3.67105 & -2.18713 & 1.38377 \\
\hline $\mathrm{H}$ & & 5.99924 & -0.29265 & 1.66034 \\
\hline $\mathrm{H}$ & & 6.06367 & -1.99987 & 1.63704 \\
\hline & C & -0.03737324 & -0.00038597 & -0.01447899 \\
\hline & C & 1.33706132 & -0.00118427 & 0.03821004 \\
\hline & C & 2.01350203 & -0.02359457 & 1.25811476 \\
\hline & C & 3.46267775 & -0.00100065 & 1.38246180 \\
\hline & C & 4.21673774 & -1.15052595 & 1.45985998 \\
\hline & $\mathrm{N}$ & 5.52810716 & -1.18094197 & 1.58984446 \\
\hline Optimized geometry: & $\mathrm{H}$ & -0.55842293 & 0.01124181 & -0.95378630 \\
\hline pimmized geonimetry. & $\mathrm{H}$ & -0.63076255 & -0.01119979 & 0.88213443 \\
\hline & $\mathrm{H}$ & 1.90624323 & 0.01274392 & -0.87396292 \\
\hline & $\mathrm{H}$ & 1.42163422 & -0.01694892 & 2.16149225 \\
\hline & $\mathrm{H}$ & 3.94410548 & 0.96269340 & 1.44464824 \\
\hline & $\mathrm{H}$ & 3.73938303 & -2.11278518 & 1.41243745 \\
\hline & $\mathrm{H}$ & 6.07201668 & -0.34734600 & 1.64636103 \\
\hline & $\mathrm{H}$ & 6.01704007 & -2.04708587 & 1.63712379 \\
\hline
\end{tabular}




$\begin{array}{rrrr}C & 0.154078240 & -0.002141697 & 0.085312898 \\ \text { C } & -0.093231597 & -0.009204196 & -0.080434864 \\ \text { C } & -0.003805728 & 0.049532881 & 0.016130191 \\ \text { C } & 0.240968157 & -0.358256184 & -0.001617127 \\ \text { C } & -0.604879940 & 0.336679215 & -0.066840124 \\ \text { N } & 0.502899376 & -0.045419846 & 0.048523609 \\ \hat{x} \text { vector: } H & -0.006488741 & 0.000899080 & -0.011806337 \\ \text { H } & -0.007206103 & -0.000922633 & 0.007429869 \\ \text { H } & 0.006603713 & -0.002644582 & 0.013898090 \\ \text { H } & -0.044823020 & -0.000380078 & -0.013847761 \\ \text { H } & -0.067853177 & 0.028398694 & 0.007384049 \\ \text { H } & -0.003148269 & 0.003110919 & 0.000772413 \\ \text { H } & -0.036635966 & -0.079373698 & -0.002290248 \\ \text { H } & -0.036476945 & 0.079722127 & -0.002614658 \\ & & & \\ & & & \\ \text { C } & -0.006929613 & 0.014402749 & 0.003836408 \\ \text { C } & 0.016240221 & 0.501295458 & 0.016501890 \\ \text { C } & 0.006206657 & -0.018649421 & -0.068673768 \\ \text { C } & -0.002303946 & -0.006354635 & 0.059098453 \\ \text { C } & -0.067984790 & 0.010724730 & 0.557215866 \\ \text { N } & 0.018418362 & -0.001791159 & -0.062857266 \\ \hat{y} \text { vector: } H & -0.000089807 & -0.054329584 & -0.000759044 \\ H & -0.000372802 & -0.010707117 & -0.000114037 \\ \text { H } & 0.001508529 & 0.003023948 & 0.001728839 \\ \text { H } & -0.009852212 & -0.446456303 & 0.003714708 \\ \text { H } & 0.041893841 & 0.008639385 & -0.461175432 \\ \text { H } & -0.000288779 & 0.000019622 & 0.004595013 \\ \text { H } & -0.000798685 & -0.001819723 & -0.001562567 \\ \text { H } & 0.004353024 & 0.002002050 & -0.051549063\end{array}$


S17 Me-PSB5 (q)
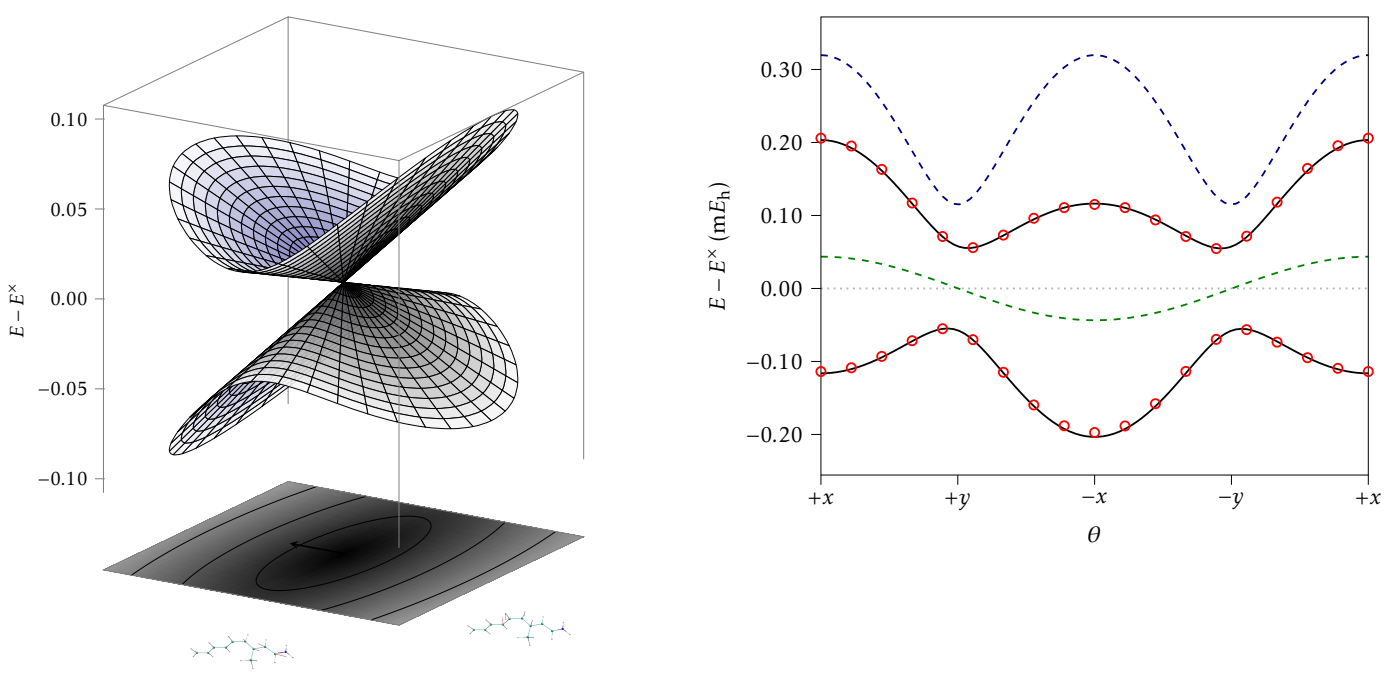

$\begin{array}{rrrr}\text { C } & 0.00000 & 0.00000 & 0.00000 \\ \text { C } & 1.35106 & 0.00000 & 0.00000 \\ \text { C } & 2.08400 & 0.00000 & 1.24497 \\ \text { C } & 3.45663 & 0.00217 & 1.25945 \\ \text { C } & 4.13348 & 0.00216 & 2.51554 \\ \text { C } & 5.48752 & -0.00272 & 2.77843 \\ \text { C } & 6.38218 & -1.10918 & 2.93576 \\ \text { C } & 7.71928 & -0.77474 & 3.19521 \\ \text { C } & 8.73868 & -1.72795 & 3.37923 \\ \text { N } & 9.98751 & -1.34344 & 3.62205 \\ \text { C } & 5.92507 & -2.51991 & 2.82993 \\ \text { H } & -0.56547 & -0.00025 & -0.92305 \\ \text { Initial geometry: } & -0.57460 & 0.00054 & 0.92020 \\ \text { H } & 1.92030 & -0.00091 & -0.93543 \\ \text { H } & 1.50555 & -0.00031 & 2.18212 \\ \text { H } & 4.00429 & 0.00135 & 0.31792 \\ \text { H } & 3.47357 & 0.00398 & 3.40544 \\ \text { H } & 5.95557 & 0.98865 & 2.88237 \\ \text { H } & 7.99332 & 0.28172 & 3.26071 \\ \text { H } & 8.51602 & -2.81202 & 3.32418 \\ \text { H } & 10.24527 & -0.37183 & 3.68228 \\ \text { H } & 10.73625 & -2.00148 & 3.75985 \\ \text { H } & 6.74237 & -3.24253 & 2.98057 \\ \text { H } & 5.17575 & -2.70071 & 3.60306 \\ \text { H } & 5.52099 & -2.67989 & 1.82860\end{array}$




\begin{tabular}{|c|c|c|c|c|c|}
\hline & C & 0.03064899 & -0.04619607 \\
\hline & & & C & 1.38532110 & -0.01265411 \\
\hline & & & C & 2.04213520 & -0.04012270 \\
\hline & & & $c$ & 3.43822726 & -0.00487288 \\
\hline & & & C & 4.05206798 & -0.03458753 \\
\hline & & & C & 5.50548658 & 0.05125323 \\
\hline & & & C & 6.37490715 & -1.00997356 \\
\hline & & & C & 7.75077452 & -0.72234092 \\
\hline & & & C & 8.73760833 & -1.68649361 \\
\hline & & & $\mathrm{N}$ & 10.01589634 & -1.42275315 \\
\hline & & & C & 5.89977322 & -2.44030830 \\
\hline & & & $\mathrm{H}$ & -0.44123266 & -0.02258004 \\
\hline \multirow{13}{*}{\multicolumn{3}{|c|}{ Optimized geometry: }} & $\mathrm{H}$ & -0.61126801 & -0.09587742 \\
\hline & & & $\mathrm{H}$ & 1.99484894 & 0.03866776 \\
\hline & & & H & 1.43438618 & -0.08866433 \\
\hline & & & H & 4.04576421 & 0.04761868 \\
\hline & & & $\mathrm{H}$ & 3.43679488 & -0.07041626 \\
\hline & & & $\mathrm{H}$ & 5.90695911 & 1.04708187 \\
\hline & & & H & 8.04782208 & 0.30955842 \\
\hline & & & H & 8.49606159 & -2.73010482 \\
\hline & & & $\mathrm{H}$ & 10.35255116 & -0.48858914 \\
\hline & & & $\mathrm{H}$ & 10.68507043 & -2.15473454 \\
\hline & & & $\mathrm{H}$ & 6.15249648 & -2.99693301 \\
\hline & & & $\mathrm{H}$ & 4.82826227 & -2.49127651 \\
\hline & & & H & 6.35322668 & -2.94200108 \\
\hline \multirow{25}{*}{$\hat{x}$ vector: } & C & \multicolumn{2}{|c|}{-0.060050883} & -0.000258712 & -0.060398664 \\
\hline & C & \multicolumn{2}{|c|}{0.016706827} & -0.002209122 & 0.064176763 \\
\hline & $\mathrm{C}$ & \multicolumn{2}{|c|}{-0.083816551} & -0.002112620 & -0.060762477 \\
\hline & C & \multicolumn{2}{|c|}{0.084507349} & 0.000913677 & 0.091152342 \\
\hline & C & \multicolumn{2}{|c|}{-0.025695741} & -0.022978921 & -0.032041493 \\
\hline & $\mathrm{C}$ & \multicolumn{2}{|c|}{-0.094541978} & 0.162535228 & 0.007035735 \\
\hline & C & \multicolumn{2}{|c|}{0.293918212} & -0.121182132 & 0.038111160 \\
\hline & C & \multicolumn{2}{|c|}{-0.393667301} & 0.235229590 & -0.047942610 \\
\hline & $\mathrm{C}$ & \multicolumn{2}{|c|}{0.579291952} & -0.175701133 & 0.073815073 \\
\hline & C & \multicolumn{2}{|c|}{-0.467423711} & -0.056629298 & -0.061976046 \\
\hline & $\mathrm{N}$ & \multicolumn{2}{|c|}{0.002650880} & -0.026532538 & 0.000346512 \\
\hline & $\mathrm{H}$ & \multicolumn{2}{|c|}{-0.004453390} & 0.000029839 & 0.002700831 \\
\hline & $\mathrm{H}$ & \multicolumn{2}{|c|}{0.002519890} & 0.000428715 & -0.000316933 \\
\hline & $\mathrm{H}$ & \multicolumn{2}{|c|}{-0.000802664} & 0.000806063 & -0.010079064 \\
\hline & $\mathrm{H}$ & \multicolumn{2}{|c|}{0.004518821} & 0.001342768 & -0.004967082 \\
\hline & $\mathrm{H}$ & \multicolumn{2}{|c|}{0.002390170} & 0.002320784 & -0.011400940 \\
\hline & $\mathrm{H}$ & \multicolumn{2}{|c|}{0.031320407} & 0.005655503 & 0.006774128 \\
\hline & $\mathrm{H}$ & \multicolumn{2}{|c|}{0.042263452} & -0.011908066 & 0.001264372 \\
\hline & H & \multicolumn{2}{|c|}{0.004582252} & -0.002479358 & 0.000072770 \\
\hline & $\mathrm{H}$ & 0.006 & 695305 & -0.002190499 & 0.000583374 \\
\hline & $\mathrm{H}$ & 0.015 & 046868 & 0.056686602 & 0.002423962 \\
\hline & $\mathrm{H}$ & 0.034 & 070071 & -0.050012124 & 0.001855695 \\
\hline & $\mathrm{H}$ & -0.004 & 403398 & -0.004627622 & 0.005110937 \\
\hline & $\mathrm{H}$ & 0.019 & 693029 & 0.015740079 & 0.000458086 \\
\hline & $\mathrm{H}$ & -0.005 & 319865 & -0.002866705 & -0.005996432 \\
\hline
\end{tabular}




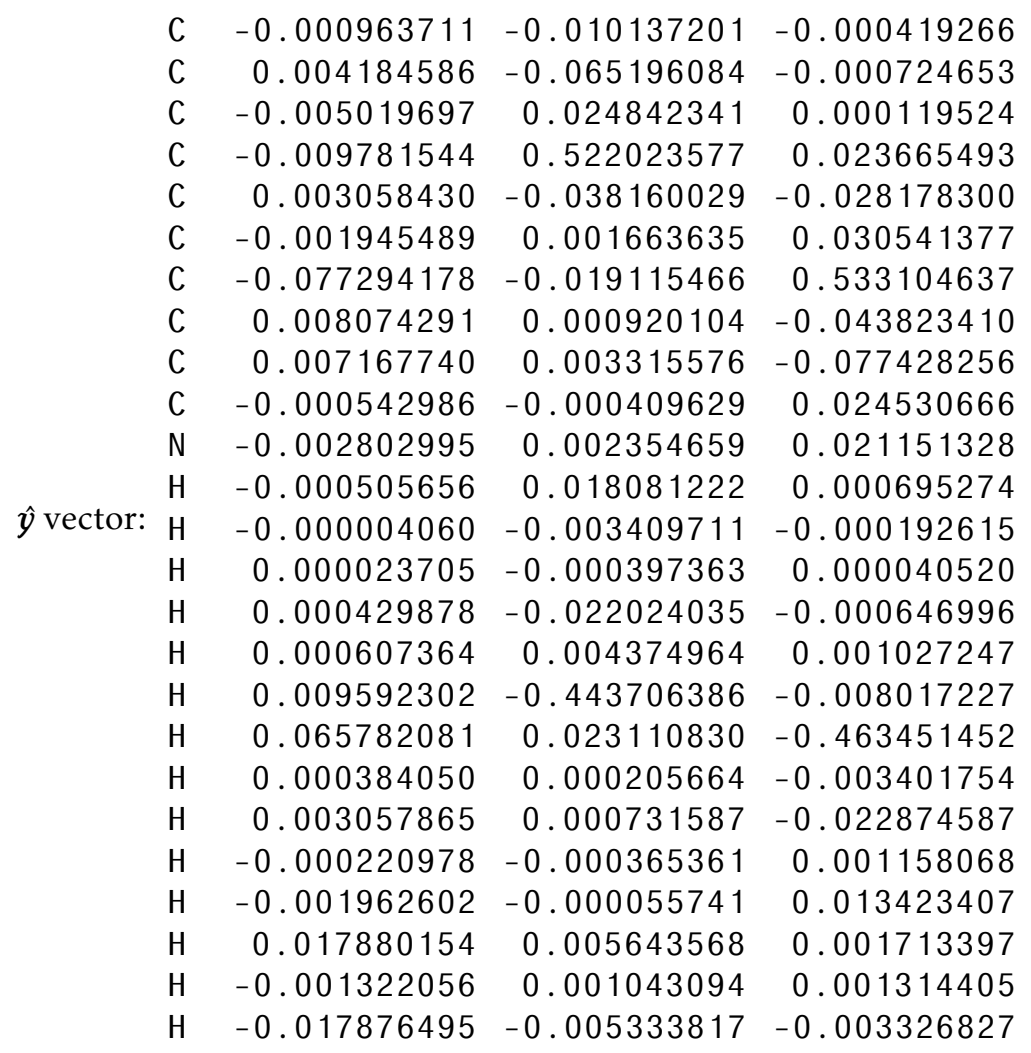




\section{S18 Stilbene ( $r)$}
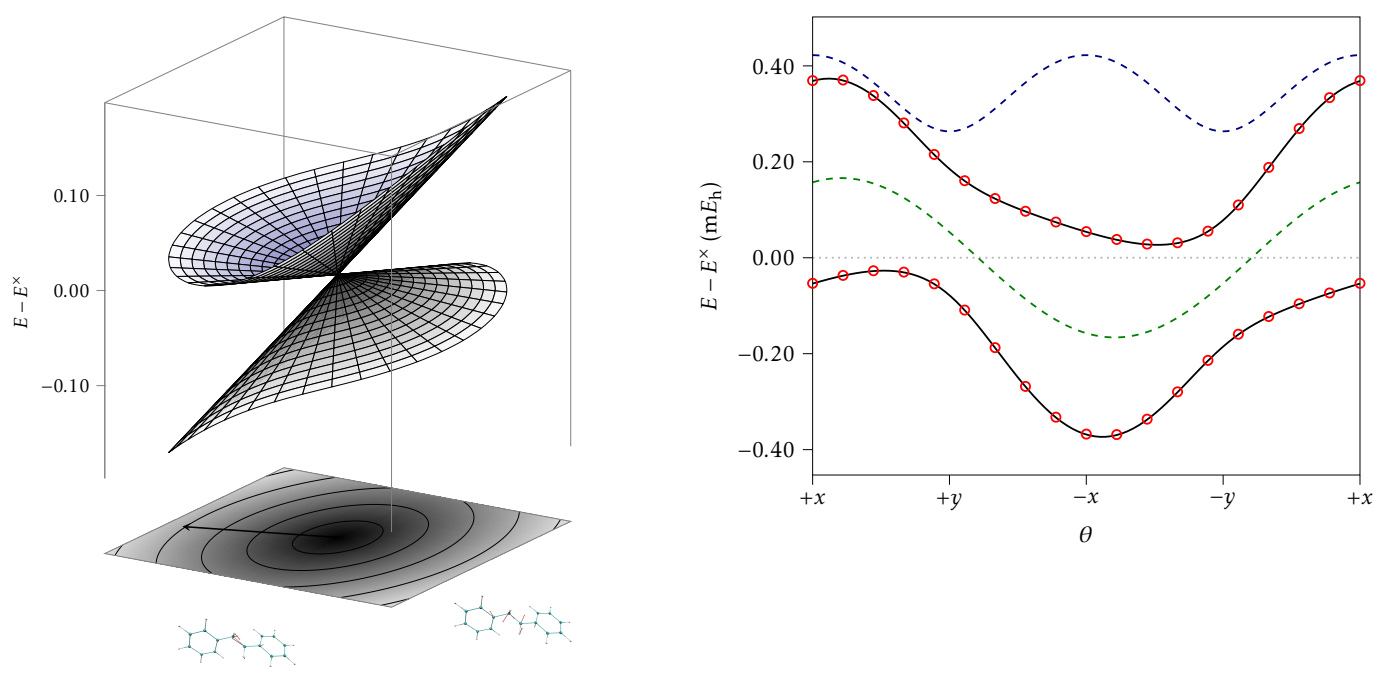

$\begin{array}{rrr}\text { C } & 1.623237 & 2.250629 \\ \text { C } & 1.146150 & 1.530120 \\ \text { C } & 1.895590 & 1.665884 \\ \text { C } & 3.048362 & 2.470947 \\ \text { C } & 3.486147 & 3.152964 \\ \text { C } & 2.783892 & 3.051287 \\ \text { C } & -0.002040 & 0.704510 \\ \text { C } & 0.002040 & -0.704510 \\ \text { C } & -1.146150 & -1.530120 \\ \text { C } & -2.493400 & -1.040480 \\ \text { C } & -3.575870 & -1.949869 \\ \text { C } & -3.348430 & -3.344490 \\ \text { C } & -2.064070 & -3.839820 \\ \text { C } & -0.966260 & -2.958320 \\ \text { H } & -0.540674 & 0.702950 \\ \text { H } & 0.950480 & -1.215370 \\ \text { H } & 1.556713 & 1.133081 \\ \text { H } & 0.039160 & -3.352490 \\ \text { H } & 3.584600 & 2.547872 \\ \text { H } & -1.888750 & -4.906320 \\ \text { H } & 4.371255 & 3.771698 \\ \text { H } & -4.192720 & -4.019989 \\ \text { H } & 3.136236 & 3.590842 \\ \text { H } & -4.588000 & -1.571849 \\ \text { H } & 1.106594 & 2.189869 \\ \text { H } & -2.693100 & 0.016510\end{array}$

1. 143715

0.000000

$-1.221484$

$-1.293724$

$-0.181011$

1. 040952

0.000000

0.000000

$-0.000000$

$-0.000000$

$-0.000000$

$-0.000000$

$-0.000000$

$-0.000000$

$-0.932946$

0.000000

$-2.097568$

$-0.000000$

$-2.228973$

$-0.000000$

$-0.234661$

$-0.000000$

1.908154

$-0.000000$

2. 085254

$-0.000000$ 


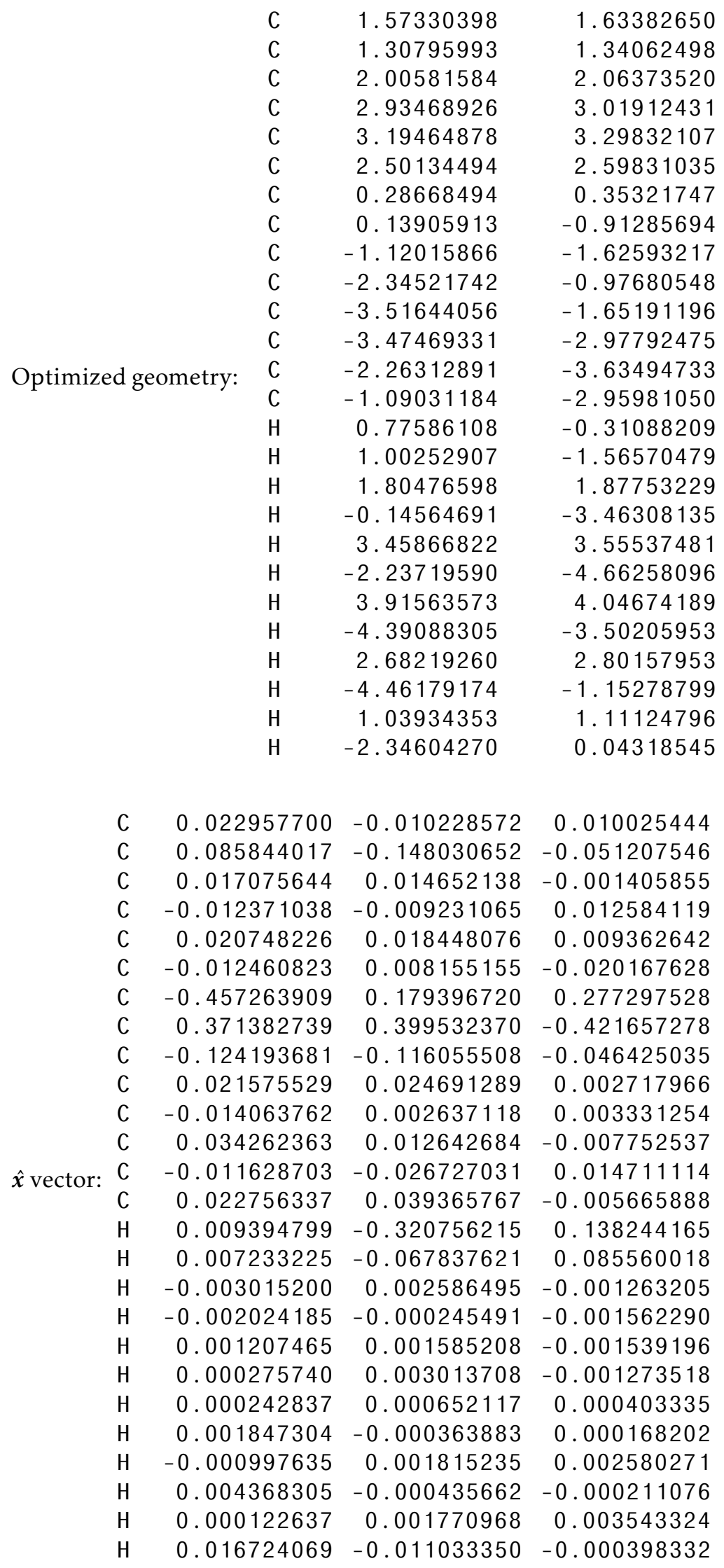




$\begin{array}{rrrr}C & -0.050692069 & -0.028429379 & -0.016674814 \\ \text { C } & -0.054749340 & 0.318934184 & -0.163272178 \\ \text { C } & 0.009403953 & -0.013028733 & 0.028803462 \\ \text { C } & -0.009541922 & -0.000115267 & 0.028406767 \\ \text { C } & 0.015497430 & 0.020210826 & 0.008295729 \\ \text { C } & 0.024174774 & -0.002215844 & -0.049086802 \\ \text { C } & -0.039875426 & -0.294637122 & 0.163262677 \\ \text { C } & -0.328578256 & 0.073301667 & -0.264472702 \\ \text { C } & -0.019742493 & 0.049730326 & 0.312496091 \\ \text { C } & -0.010764142 & -0.011721849 & 0.001310645 \\ \text { C } & 0.008974118 & 0.022710018 & 0.012963584 \\ \text { C } & 0.012746894 & -0.013168715 & 0.005625775 \\ \hat{y} \text { vector: C } & -0.018192815 & -0.006675686 & 0.000048637 \\ \text { C } & 0.007979062 & 0.017395677 & -0.009527601 \\ \text { H } & 0.445554340 & -0.004602359 & 0.342667518 \\ \text { H } & -0.005919048 & -0.113538360 & -0.358279444 \\ \text { H } & 0.008329981 & 0.001487683 & -0.004065923 \\ \text { H } & 0.001338015 & -0.001746630 & -0.005463084 \\ \text { H } & -0.000402718 & 0.000610411 & -0.001656196 \\ \text { H } & -0.000817822 & 0.000355594 & 0.000675063 \\ \text { H } & 0.002042523 & -0.002502283 & -0.000155500 \\ \text { H } & -0.000112456 & -0.003088334 & -0.009722249 \\ \text { H } & -0.002951932 & -0.004842830 & 0.000741564 \\ \text { H } & 0.000112636 & 0.000142937 & 0.000859374 \\ \text { H } & 0.003236098 & 0.000738751 & 0.001071822 \\ \text { H } & 0.002950613 & -0.005304683 & -0.024852215\end{array}$




\section{S19 GFP chromophore (s)}

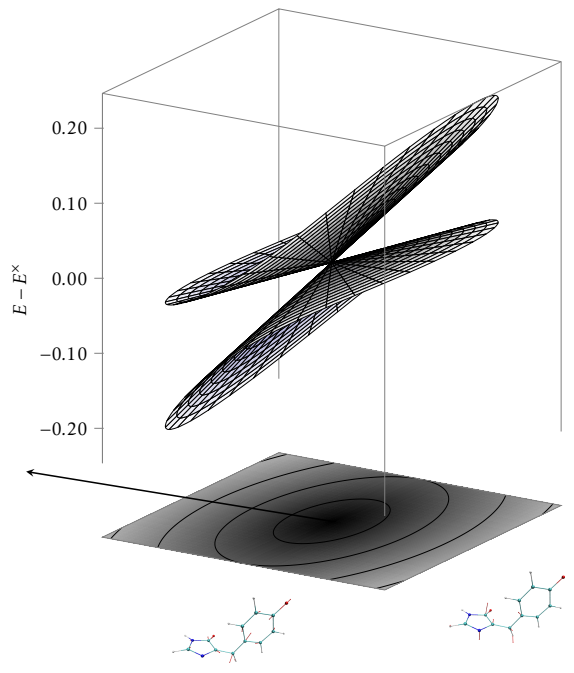

$\begin{array}{rrr}\text { N } & 2.012911 & -0.503601 \\ \text { C } & 1.887513 & -0.778717 \\ \text { C } & 3.262207 & -0.849987 \\ \text { N } & 4.060820 & -0.606501 \\ \text { C } & 3.269706 & -0.400202 \\ \text { C } & 0.717870 & -0.958090 \\ \text { C } & 0.061864 & 0.188027 \\ \text { Initial geometry: } & -1.069416 & -0.005322 \\ \text { C } & -1.679313 & 1.071395 \\ \text { C } & -1.177950 & 2.353502 \\ \text { C } & -0.059278 & 2.552301 \\ \text { O } & 0.562500 & 1.483032 \\ \text { O } & 3.786228 & 3.415366 \\ \text { H } & -2.550485 & -1.079564 \\ \text { H } & -1.459419 & -1.000406 \\ \text { H } & 0.689908 & -1.927259 \\ \text { H } & 5.030425 & -0.582344 \\ \text { H } & 3.643761 & -0.183206 \\ \text { H } & 1.433506 & 1.638186 \\ \text { H } & 0.325305 & 3.550193\end{array}$

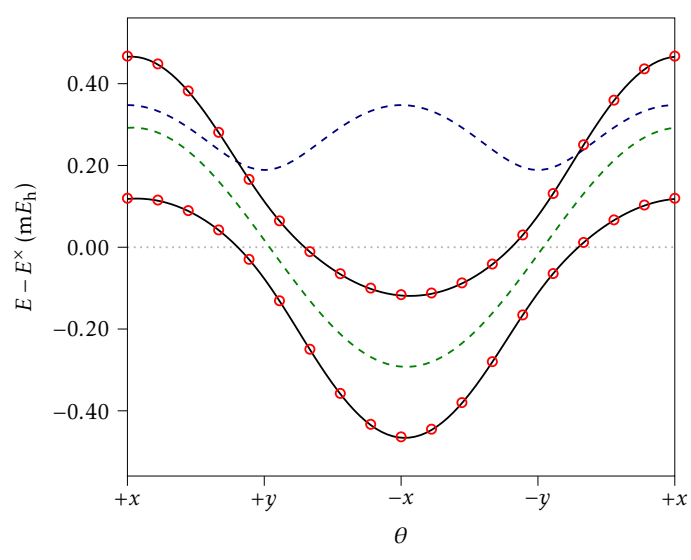

$-0.901987$

0.431382

0.955474

-0. 107162

$-1.202152$

1. 137160

1. 771042

2.576565

3. 166821

2. 967914

2. 165764

1.574430

3.554520

2. 101163

3. 787744

2. 732050

1.612860

$-0.094890$

$-2.191818$

0.954997

2. 015012 


\begin{tabular}{|c|c|c|c|c|c|}
\hline & $\mathrm{N}$ & 2.13648259 & -0.58693908 \\
\hline & & & C & 1.91696302 & -0.78388300 \\
\hline & & & $\mathrm{C}$ & 3.25249882 & -0.86695518 \\
\hline & & & $\mathrm{N}$ & 4.14629211 & -0.65824130 \\
\hline & & & C & 3.40905041 & -0.50953587 \\
\hline & & & C & 0.61590375 & -0.89434350 \\
\hline & & & C & 0.04497302 & 0.22464184 \\
\hline & & & C & -0.98411773 & 0.02821507 \\
\hline & & & c & -1.58712656 & 1.05437808 \\
\hline & & & C & -1.21910081 & 2.42658650 \\
\hline \multirow{11}{*}{\multicolumn{3}{|c|}{ Optimized geometry: }} & c & -0.17425670 & 2.60990755 \\
\hline & & & C & 0.40960970 & 1.56391108 \\
\hline & & & 0 & -1.76085333 & 3.37123719 \\
\hline & & & 0 & 3.55961808 & -1.09090747 \\
\hline & & & $\mathrm{H}$ & -2.37079041 & 0.84591035 \\
\hline & & & H & -1.31237810 & -0.98223129 \\
\hline & & & $\mathrm{H}$ & 0.37067098 & -1.89037619 \\
\hline & & & $\mathrm{H}$ & 5.13374337 & -0.63774312 \\
\hline & & & H & 3.87344184 & -0.34069946 \\
\hline & & & H & 1.17545717 & 1.79744127 \\
\hline & & & $\mathrm{H}$ & 0.14586379 & 3.62019555 \\
\hline \multirow{21}{*}{$\hat{x}$ vector: } & $\mathrm{N}$ & \multicolumn{2}{|c|}{-0.062386107} & -0.002447767 & 0.058886336 \\
\hline & C & \multicolumn{2}{|c|}{-0.246545288} & -0.010573773 & -0.026334928 \\
\hline & C & \multicolumn{2}{|c|}{0.280733449} & -0.062113771 & 0.274226932 \\
\hline & $\mathrm{N}$ & \multicolumn{2}{|c|}{0.083110942} & 0.019748301 & -0.075619341 \\
\hline & C & \multicolumn{2}{|c|}{0.057427286} & -0.017002104 & 0.159910904 \\
\hline & C & \multicolumn{2}{|c|}{-0.054071619} & -0.286084021 & -0.295667523 \\
\hline & C & \multicolumn{2}{|c|}{-0.105596089} & 0.349259707 & 0.210358374 \\
\hline & C & \multicolumn{2}{|c|}{0.173123307} & -0.046458415 & -0.145611386 \\
\hline & c & \multicolumn{2}{|c|}{0.001738204} & 0.180093930 & 0.015083179 \\
\hline & C & \multicolumn{2}{|c|}{0.087232162} & -0.168794032 & -0.106608281 \\
\hline & $\mathrm{C}$ & \multicolumn{2}{|c|}{-0.116262432} & 0.054850870 & 0.117377093 \\
\hline & $\mathrm{C}$ & \multicolumn{2}{|c|}{-0.026319908} & -0.247342210 & -0.000809314 \\
\hline & 0 & \multicolumn{2}{|c|}{-0.084980776} & 0.157740875 & 0.100308758 \\
\hline & 0 & \multicolumn{2}{|c|}{-0.103196023} & 0.051368705 & -0.297384364 \\
\hline & $\mathrm{H}$ & \multicolumn{2}{|c|}{-0.006443393} & -0.000585524 & 0.002613747 \\
\hline & $\mathrm{H}$ & \multicolumn{2}{|c|}{-0.001481480} & -0.009744627 & 0.001195071 \\
\hline & $\mathrm{H}$ & \multicolumn{2}{|c|}{0.100466749} & 0.009053307 & 0.026322103 \\
\hline & $\mathrm{H}$ & \multicolumn{2}{|c|}{0.011583417} & 0.003522426 & -0.007570492 \\
\hline & $\mathrm{H}$ & \multicolumn{2}{|c|}{0.001352967} & -0.002232706 & 0.001490123 \\
\hline & $\mathrm{H}$ & \multicolumn{2}{|c|}{0.009225160} & 0.019261223 & -0.011932721 \\
\hline & $\mathrm{H}$ & \multicolumn{2}{|c|}{0.001289469} & 0.008479606 & -0.000234269 \\
\hline
\end{tabular}




$\begin{array}{rrrr}\text { N } & -0.022419825 & -0.449125330 & -0.041687146 \\ \mathrm{C} & 0.049184369 & 0.192112138 & 0.111398218 \\ \mathrm{C} & 0.002436801 & 0.582422904 & 0.149772162 \\ \mathrm{~N} & 0.024187835 & -0.133636753 & -0.059643264 \\ \mathrm{C} & 0.032381123 & -0.020315015 & 0.037164670 \\ \mathrm{C} & -0.178149623 & -0.012832422 & -0.068847389 \\ \mathrm{C} & 0.276559042 & -0.078706228 & 0.219199033 \\ \mathrm{C} & 0.011984778 & 0.019038284 & 0.078711404 \\ \mathrm{C} & -0.011301884 & -0.035821760 & -0.022591254 \\ \mathrm{C} & 0.055694731 & 0.025630309 & 0.003933949 \\ \hat{y} \text { vector: } & -0.012129507 & 0.015714026 & 0.034351822 \\ \mathrm{C} & 0.023140265 & -0.028767387 & -0.020397507 \\ \text { O } & -0.014879113 & -0.004694055 & -0.003564679 \\ \mathrm{O} & 0.013293206 & -0.101116621 & -0.057957777 \\ \mathrm{H} & -0.011548602 & 0.001477060 & -0.010193938 \\ \mathrm{H} & 0.000090180 & -0.000293686 & 0.001163407 \\ \mathrm{H} & -0.211729823 & -0.034936642 & -0.331648689 \\ \mathrm{H} & 0.002014000 & 0.005768260 & -0.001994158 \\ \mathrm{H} & -0.002660957 & 0.059411505 & 0.008838226 \\ \mathrm{H} & -0.016114141 & -0.002836986 & -0.016814924 \\ \mathrm{H} & -0.010032855 & 0.001508400 & -0.009192164\end{array}$

